Open Access

\title{
Effects of whole-body vibration on proxies of muscle strength in old adults: a systematic review and meta-analysis on the role of physical capacity level
}

Slavko Rogan", Eling D. de Bruin ${ }^{1 *}$, Lorenz Radlinger ${ }^{1}$, Christine Joehr ${ }^{1}$, Christa Wyss' ${ }^{1}$, Neil-Jerome Stuck ${ }^{1}$, Yvonne Bruelhart ${ }^{1}$, Rob A. de Bie ${ }^{1}$ and Roger Hilfiker ${ }^{1}$

\begin{abstract}
Background: Dynapenia (age-associated loss of muscle strength not caused by neurologic or muscular diseases) and functional limitations (e.g. climbing stairs, chair rising) are important problems in elderly persons. Whole body vibration, used as an adjunct to classical resistance training or even as a stand-alone alternative, might help to reduce these problems. Its value might be highest in elderly persons with very low function, where whole body vibration can be used as a skilling up training until more conventional exercise types are possible. This systematic review and meta-analysis summarized the current evidence for whole-body vibration interventions on isometric maximum voluntary contraction, dynamic strength, power, rate of force development and functional strength in elderly categorised in different subgroups based on function levels.

Methods: An extensive literature search was carried out in February 2014 and repeated in February 2015 at PubMed, Cochrane Central Register of Controlled Trials, Physiotherapy Evidence Database and CINAHL electronic databases. The International Clinical Trials Registry Platform from the World Health Organization was also searched. Randomized controlled trials measuring isometric maximum voluntary contraction, dynamic strength, power, rate of force development and functional strength in studies using WBV intervention in 65 years or older elderly individuals were included. The methodological quality of included studies was assessed using the Cochrane Collaboration's tool for assessing Risk of Bias. Studies were classified based on the level of physical capacitiy of the participants as "Go-Go", "Slow-Go" or "No-Go". Data were pooled using a random effects model.

(Continued on next page)
\end{abstract}

\footnotetext{
*Correspondence: eling.debruin@hest.ethz.ch

'Bern University of Applied Sciences, Discipline Physiotherapy, Bern, Switzerland

Full list of author information is available at the end of the article
}

\section{Biomed Central}

(c) 2015 Rogan et al. Open Access This article is distributed under the terms of the Creative Commons Attribution 4.0 International License (http://creativecommons.org/licenses/by/4.0/), which permits unrestricted use, distribution, and reproduction in any medium, provided you give appropriate credit to the original author(s) and the source, provide a link to the Creative Commons license, and indicate if changes were made. The Creative Commons Public Domain Dedication waiver (http://creativecommons.org/publicdomain/zero/1.0/) applies to the data made available in this article, unless otherwise stated. 
(Continued from previous page)

Results: Thirty-eigth articles of moderate methodological quality were included. The vibration modes for sinusoidal vertical whole-body vibration was between 25 and $40 \mathrm{~Hz}$, the amplitude varied from 2 to $4 \mathrm{~mm}$. Sinusoidal sidealternating -whole-body vibration revealed frequencies from 2.5 to $35 \mathrm{~Hz}$ with amplitudes ranging from 0.05 to $12 \mathrm{~mm}$. Stochastic resonance whole-body vibration used frequencies between 3 and $6 \mathrm{~Hz}$. Effect sizes in Go-Go were moderate after vertical sinusoidal Whole-body vibration compared to non-training control groups for isometric maximum voluntary contraction with effect size $0.48(95 \% \mathrm{Cl} 0.33$ to 0.63$)$ and for Dynamic Strength with effect size 0.47 (95\% Cl 0.06 to 0.88). Side-alternating sinusoidal whole body vibration showed moderate effect sizes with 0.69 ( $95 \% \mathrm{Cl} 0.32$ to 1.06) for isometric maximum voluntary contraction, 0.50 (95\% Cl 0.07 to 0.92 ) for power, 0.40 (95\% Cl 0.16 to 0.64) for Rate of Force Development and 0.42 ( $95 \%$ Cl 0.13 to 0.71 ) for Functional Strength compared to non-exercise control. The analysis for Slow-Go showed for stochastic resonance whole-body vibration and Functional Strength an effect size of $0.97(95 \% \mathrm{Cl}-0.07$ to 2.00) compared to non-exercise control in one study. No-Go showed for stochastic resonance whole-body vibration a moderate effect size with $0.50(95 \% \mathrm{Cl}$ -0.32 to 1.33 ) for Functional Strength compared to non-exercise control.

Conclusions: Whole-body vibration shows beneficial effects, mainly in the No-Go group elderly compared to nontraining control and conventional strength training groups. The results suggest that WBV can be used as a skillingup exercise in participants not able to perform standard exercises. Further studies with the various types of WBV in various sub-populations of elderly persons are needed to determine the most effective vibration modes.

Trial registration: Registration number: CRD42013006489.

Keywords: WBV, Isometric maximum voluntary contraction, Dynamic maximum voluntary contraction, Power, Rate of force development, Functional strength

\section{Background}

Aging is associated with a decrease of muscle strength and power [1-3]. The term dynapenia, coined by Manini and Clark [3-5], best describes the condition of decreased muscle strength and power instead of the term sarcopenia. The latter only refers to an age-related loss in skeletal muscle mass. Muscle weakness is related to falls, lower walking speed, functional limitation, a decrease in mobility, and disability [6-8]. In this context, the elderly are viewed as a group of people in need [9]. When physical functioning is concerned there often is a mismatch between chronological age and biological age. Chronological age is not necesarilly related to physical capabilities. For this reason, a classification of elderly based on physical abilities; e.g. physical and mental functions is more appropriate.

Zeyfang and Braun [10] classified older adults as "being an independent person" (Go-Go); "being a needy person with a slight handicap" (Slow-Go); and "being a person in need of care with severe functional limitation" (No-Go). The need for care may be defined as depending permanently on assistance (No-Go) or depending on support in everyday activities such as dressing, body care, eating, using the toilet, mobility, and planning the day (Slow-Go) [11].

The ability of elderly individuals to perform basic activities of daily life is crucial for their ability to exist independently [12]. To improve and/or enable performance of basic activities of daily life, exercise programs are indicated. Sensorimotor training and resistance exercises are effective methods to increase muscle mass and strength in the elderly [13]. Whole-body vibration (WBV) can be used as a sensorimotor training regimen. The impact of WBV on the body is low according to indicators such as blood pressure, heart rate, lactate, and $\mathrm{O}_{2}$ uptake [14-16]. Systematic reviews concluded that, compared to more demanding interventions, WBV might be a safer and less fatiguing type of exercise [17] with a beneficial effect on movement skills [18].

Three types of WBV are used based on the amount of vibrating plates $[18,19]$. Sinusoidal vertical whole-body vibration (SV-WBV) and sinusoidal side-alternating whole-body vibration (SS-WBV) use a single vibrating platform, whereas stochastic resonance whole-body vibration (SR-WBV) expects the trainees to stand on two platforms. During sinusoidal WBV the participants stand on a platform that vibrates vertically (SV-WBV) or to the side alternating (SS-WBV) with a high frequency between 20 and $50 \mathrm{~Hz}$ and an amplitude between 2 and $14 \mathrm{~mm}$ [20]. SR-WBV vibrates with frequencies between 1 and $12 \mathrm{~Hz}$ and an amplitude between 3 and $6 \mathrm{~mm}$ while the feet of the participants are placed on two independent powered and stochastic vibrating platforms [20].

In recent years, WBV has been introduced as a training method to improve muscle power and strength [21-23]. Several systematic reviews $[24,25]$ report on strengthrelated outcomes. However, no review has considered muscle strength related outcomes in a comparison of WBV against non-exercising control or conventional exercise groups and no review evaluated the effects separately 
for groups differing in initial levels of physical functioning, e.g. the three groups "Go-Go, Slow-Go and No-Go". This is of relevance, however, since training principles would let us expect that those with the lowest level of fitness have greatest room for improvement. In other words, improvement in the outcome of interest will be greatest in those with lower initial values [26]. Furthermore, no review includes stochastic resonance WBV.

For clinicians, a systematic overview about the relevance and indication for application of SV-WBV, SSWBV or SR-WBV and how it might be applied for Go-Go, Slow-Go and No-Go elderly individuals is lacking. Therefore, the aims of this systematic review are to provide 1) an overview of the current studies on WBV, 2) to determine the effects of WBV on strength or power in Go-Go, Slow-Go and No-Go elderly individuals and 3) give recommendations on available evidence for practical use. We hypothesized that WBV differently effects on measures of strength and power in Go-Go, Slow-Go and No-Go.

\section{Methods}

\section{Data sources and searches}

Inclusion criteria and analysis methods were developed and documented in a protocol prior to the current review. Included were elderly over 65 years of age; excluded were Geriatric diseases (Parkinson disease, Stroke, Multiple sclerosis), studies applying electrical current vibration or vibration with shoe insoles. More detailed information on the protocol including a link to the search strategy can be found on http://www.crd.york.ac.uk/PROSPERO/display_record.asp?ID=CRD42013006489 (PROSPERO registration number 2013:CRD42013006489). This systematic review and meta-analysis followed the PRISMA guidelines [27].

A first literature search of electronic databases was repeatedly carried out from January 2013 to February 2014 in the PubMed, CENTRAL (Cochrane Central Register of Controlled Trials), Physiotherapy Evidence Database (PEDro) and CINAHL electronic databases. The International Clinical Trials Registry Platform from the World Health Organization (WHO) was also searched. In February 2015 the search was repeated shortly before submission of the manuscript to ensure inclusion of most recent relevant material in the review. Additionally, a manual search of the reference lists of retrieved publications was conducted. English and German language restrictions were imposed upon the search.

\section{Systematic search}

The following keywords and combinations according to the PICO-model [28] were used in the search strategy:

Population: elderly, aged, dwelling home, nursing home, human research.
Intervention: Whole Body Vibration, WBV, noise, random vibration, $\mathrm{RCT}$.

Comparator: WBV against control intervention

(non-exercise or exercise on a level too low to effect on muscle [29]), WBV against conventional strength training intervention.

Outcome: strength, maximal voluntary contraction, power, rate of force development, performance, falls. Our search terms are detailed in Additional file 1.

Based on the four PICO components, a final question was stated as: For an older adult with diminishing physical capacity, will whole body vibration (WBV) exercise as compared to non- or conventionally exercising older adults improve muscle strength and/or power?

The following aspects were operationalized: 1) assessment of the quality and internal validity of the studies reviewed; 2) description of the assessments used to document the effect of WBV on isometric maximal voluntary contraction (IMVC), dynamic strength (DS), power, rate of force development (RFD) and functional strength (FS); 3) composition of the WBV training parameters; and 4) conclusion about clinical relevance in general.

\section{Study selection}

Five independent reviewers (CJ, CW, NJS, SR, RH) screened the titles and abstracts for eligibility. They screened for randomized controlled trials (RCTs) measuring maximal voluntary contraction, power and rate of force development in studies using WBV intervention in elderly individuals (mean age at least 65 years). Full text articles in English or German were eligible for inclusion. Healthy elderly participants and all clinical outcome measures of IMVC, DS, power, RFD, and FS were included in this review. Studies describing vibrations applied by electrical current or vibrating insoles, and patient series were excluded.

In the event of missing data, additional information was requested from the corresponding authors in order to include these data in our meta-analysis.

\section{Data extraction}

In addition, general characteristics of the studies were extracted. Five authors (CJ, CW, NJS, SR, RH) independently abstracted the following information from each of the studies included in this review: 1) design and sample; 2 ) inclusion criteria; 3) training parameters (i. e. duration, frequency, intensity of WBV); 4) type of vibration plate; 5) change in strength, power, RFD; 6) conclusions of the studies and statistical significance.

\section{Methodological quality assessment of studies}

The methodological quality of the included articles was rated with the "Cochrane Collaboration tool for 
assessing risk of bias" (RoB) [30] to assess the risk of overor under-estimating the effects of an intervention [31].

Nine items, with each having three rating categories, were scored and divided into six domains of bias (Fig. 2): (1) low ROB, (2) unclear ROB and (3) high ROB. Rating (1) is unlikely to alter the results significantly, (2) raises some doubt about the results and (3) seriously weakens confidence in the results. With insufficient information on an item, the score given was "high risk". The arbitration of a third reviewer was used in the event of any disagreement between the reviewers $(\mathrm{YB}, \mathrm{RH})$ for both ratings.

\section{Data synthesis and analysis}

Most outcomes of interest were presented as continuous data (mean values and SD or mean changes). For the meta-analysis of the present study the standardized mean difference (SMD) and $95 \%$ confidence interval (CIs) of the post-intervention values or changes in scores were used for all comparisons. SMDs were pooled with a random effects model. The magnitude of the effect sizes for the between groups comparisons, calculated by SMDs are interpreted as follows: an effect size (d) around 0.2 indicates a small effect size, around 0.5 a medium effect size, and around 0.8 a large effect size [32]. If only one study was identified or data were not presented in a format that allowed inclusion in the dataset, results of individual studies are presented. If studies reported more than one IMVC, DS, power or RFD, and FS; then we only extracted the first outcome data on a hierarchy of outcomes:

IMVC: 1 . isometric knee extension, 2. isometric hip extension, 3. isometric leg press; DS: 1. dynamic leg press, 2. dynamic knee extension (lowest speed), 3. dynamic hip extension; Power: 1 . leg press, 2. knee extension; RFD: 1 . counter movement jump, 2. squat jump, 3. leg press,; FS: 1. chair rise time, 2. chair rise repetition, 3. chair rise power, 4. stair climb, 5. wall squat. Subgroup analyses were undertaken to assess the effects of WBV on IMVC, DS, power, RFD and FS in Go-Go, Slow-Go and No-Go.

Heterogeneity was assessed by forest plots and the $\mathrm{I}^{2}$ statistics. Values $>25 \%$ indicate small, $>50 \%$ middle and $>75 \%$ considerable heterogeneity [33]. All other information was summarized and analysed qualitatively. Stata (version 13) was used for all meta-analyses.

\section{Results}

\section{Study selection}

The literature search yielded 1383 studies (PubMed $n=394$, Central $n=163$, Cinahl $=446$, Embase $=174$, Pedro $n=144$, ScienceDirect $n=29$, WHO International
Clinical Trials Registry Platform $n=33$ ). After identification of 367 duplicates, 1016 titles and abstracts were screened. Seventy-nine studies remained for further full-text analysis. Subsequently, 41 studies were excluded because they did not address strength, power or rate of force development or included participants with diseases. Finally, 38 full text papers [20, 23, 34-64] were included for this review and 37 were used for the meta-analysis (Fig. 1).

\section{Study characteristics}

The major characteristics of the included studies are summarized in Table 1 . Three papers investigated the effects on force by vertical and side-alternating sinusoidal WBV $[35,46,66]$. Five studies included more than 100 participants. Leung et al. [66] $(n=596)$, Boegarts et al. [38] $(n=180)$, Kemmler et al. [44] $(n=151)$ von Stengel [59] $(n=151)$ and Sitjà-Rabert et al. [64] $(n=117)$. The other included studies had a small sample size of less than 100 participants. The used strength outcomes varied across all included studies.

Table 2 shows the training parameters. All authors prescribed two to three WBV sessions per week. Intervention duration of six trials were lasting more than 1 year [37, 38, 44, 46, 59, 67]. The duration of ten trials $[35,36,42,43,50,55-58,53]$ was between 6 months and 1 year. The other trials lasted less than 6 months while one study examined strength effects immediately after a single WBV intervention [20]. The training parameters such as amplitude, frequency and sets of WBV varied across all studies investigating sinusoidal WBV. In contrast, studies with SR-WBV were more homogenous.

\section{Study methodological quality}

All studies included in Fig. 2 were at risk of bias according to the "Cochrane Collaboration's tool for assessing risk of bias". Most studies lacked allocation concealment, blinding, and presented incomplete data.

\section{Meta-analysis}

For the meta-analysis 37 studies were included and data were available for IMVC, DS, power, RFD or FS outcome measurements. The effect sizes for these outcomes are summarized in Figs. 3, 4, 5, 6, 7, 8, 9, 10, 11, 12 and 13.

\section{Isometric maximal voluntary contraction: WBV vs. non- exercise control group}

Thirteen studies [20, 23, 36-38, 45, 46, 48, 53, 57-59, 67], including 1468 participants, reported data contributing to the comparison WBV vs. non-exercise control group, one study had two WBV-arms (vertical and side-alternating) and one control arm [46] (the number of participants in the control groups was cut in half to obtain correct numbers for the pooled analysis). The pooled overall SMD was 


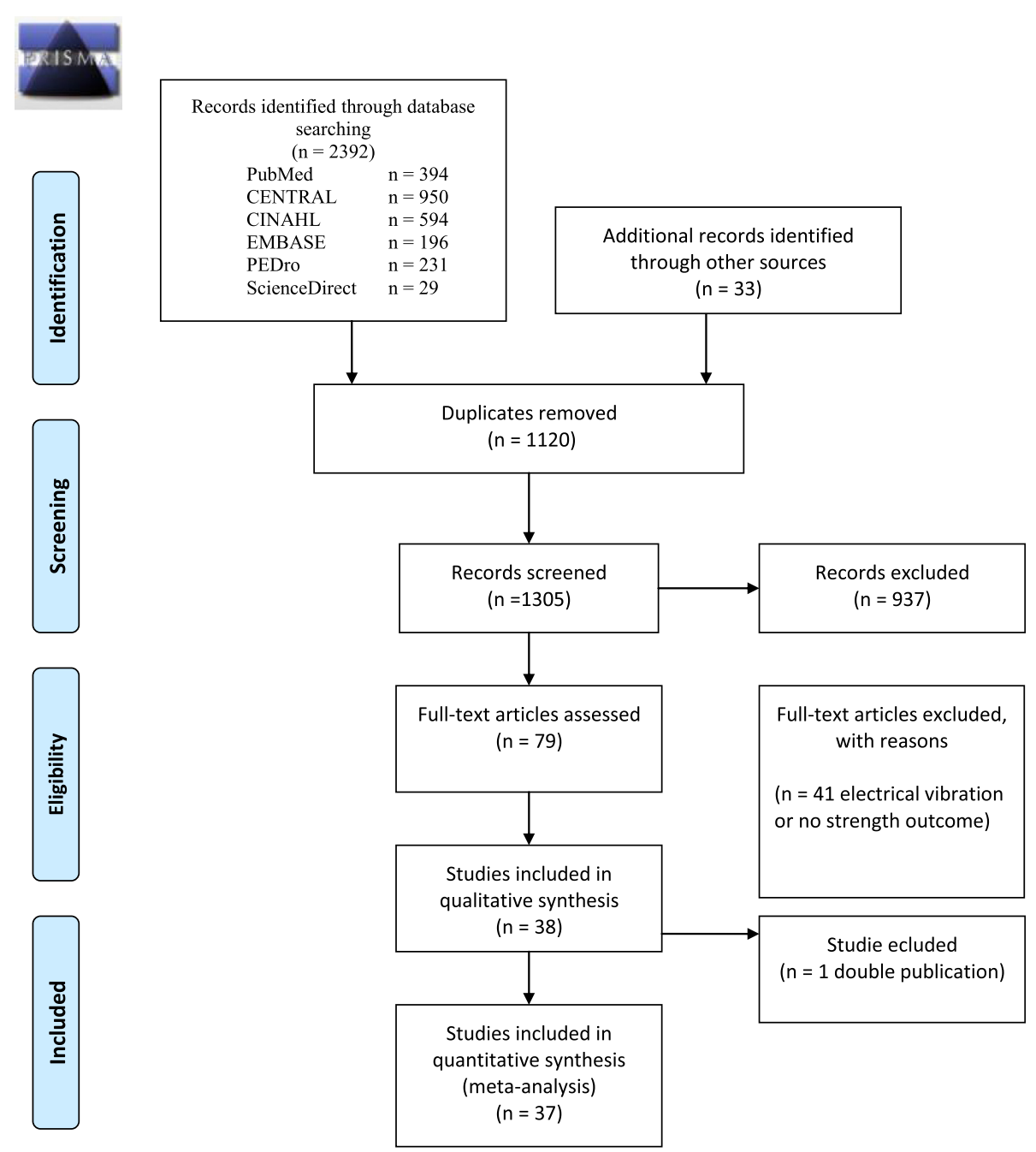

Fig. 1 Flow diagram

0.44 (95 \% CI 0.30 to 0.58 ) in favor of WBV with low heterogeneity, $\mathrm{I}^{2} 25.9 \%(p=0.176)$.

\section{Go-Go}

The subgroup analysis for SV-WBV-Go-Go showed a SMD of 0.48 (95\% CI 0.33 to 0.63 ) with a low heterogeneity $\left(\mathrm{I}^{2} 10.8 \%(p=0.346)\right)$; and for SS-WBV-Go-Go a SMD of 0.69 (95\% CI 0.32 to 1.06 ) with a low heterogeneity, $\mathrm{I}^{2} 9.8 \%(p=0.292)$.

\section{Slow-Go}

The subgroup analysis for SV-WBV-Slow-Go revealed a SMD of 0.14 (95\% CI -0.13 to 0.41 ) with a low heterogeneity, $\mathrm{I}^{2} 5.6 \%(p=0.303)$.

\section{No-Go}

The subgroup analysis for SR-WBV-No-Go showed a SMD of 0.27 (95 \% CI -0.34 to 0.88 ) in favour of SRWBV with no heterogeneity, $\mathrm{I}^{2} 0.0 \%(p=0.995)$ (Fig. 3$)$.
Isometric maximal voluntary contraction: WBV vs. exercise group

For the comparison WBV versus conventional exercise eight studies [37, 38, 45, 53, 58-60, 68] contributed 460 participants. The pooled overall SMD was 0.01 (95\% CI -0.21 to 0.22 ) with a low heterogeneity, $\mathrm{I}^{2}=$ $21.9 \%(p=0.255)$.

\section{Go-Go}

The subgroup analysis for SV-WBV-Go-Go showed a SMD of -0.16 (95\% CI -0.38 to 0.06 ) with a low heterogeneity, $\mathrm{I}^{2} 0.0 \%(p=0.966)$; SS-WBV-Go-Go showed an SMD of 0.24 (95 \% CI -0.17 to 0.65 ) in favour of SSWBV.

\section{Slow-Go}

The subgroup analysis for SS-WBV-Slow-Go showed a SMD of -0.04 (95\% CI -0.92 to 0.84 ) in favour of conventional exercise. 
Table 1 Study characteristics of the included studies

\begin{tabular}{|c|c|c|c|c|c|}
\hline Study & $\begin{array}{l}\text { Participants ( } N \text {, sex distribution); } \\
\text { mean age }( \pm S D)\end{array}$ & Protocol exercise & Outcome measures & $\begin{array}{l}\text { Within GE } \\
\text { (yes/no) }\end{array}$ & $\begin{array}{l}\text { Between GE } \\
\text { (yes/no) }\end{array}$ \\
\hline \multicolumn{6}{|l|}{ Vertical sinusoidal vibration } \\
\hline \multirow[t]{2}{*}{ Amaral et al. [78] } & WBV: 9 q; $76.6( \pm 11.8)$ & WBV: isometric squat & \multirow[t]{2}{*}{ FS: CR over $30 \mathrm{~s}$} & \multirow[t]{2}{*}{ No } & \multirow[t]{2}{*}{ No } \\
\hline & Con: 9 \&; $78.6( \pm 10.4)$ & Con: daily activities routines & & & \\
\hline \multirow[t]{2}{*}{ Álvarez- Barbosa et al. [62] } & WBV: 15,12 ; $84.0( \pm 3.0)$ & $\begin{array}{l}\text { WBV: dynamic exercise lunge, squat, } \\
\text { calf raises, left and right pivot in a } \\
\text { front and lateral position, step up } \\
\text { and down. }\end{array}$ & \multirow[t]{2}{*}{ FS: CR over $30 \mathrm{~s}$} & \multirow[t]{2}{*}{ Yes } & \multirow[t]{2}{*}{ Yes } \\
\hline & Con: 15,11 \%; $86.0( \pm 7.5)$ & Con: no change lifestyle & & & \\
\hline \multirow[t]{3}{*}{ Bautmans et al. [34] } & \multirow[t]{2}{*}{ WBV: 10,8 \%; $76.6( \pm 11.8)$} & \multirow{3}{*}{$\begin{array}{l}\text { WBV: static position exercises (lunge } \\
\text { squats, squats, deep squats, wide } \\
\text { stance squats, calves, calves deep) } \\
\text { druing WBV }\end{array}$} & DS: (N) & \multirow[t]{3}{*}{ Yes } & \multirow[t]{3}{*}{ NO } \\
\hline & & & Power: (W), work (J) & & \\
\hline & Sham: 11, 6 q; $78.6( \pm 10.4)$ & & RFD: $(\mathrm{N} / \mathrm{s})$ at 40 and $60 \mathrm{~cm} / \mathrm{s}$ & & \\
\hline \multirow[t]{2}{*}{ Beck et al. [35] } & WBV: 15 ; ; $68.5( \pm 8.6)$ & Pos: full extension & \multirow{2}{*}{$\begin{array}{l}\text { FS: wall squat with dominant leg } \\
\text { and non dominant leg \& CR over } \\
\text { five repetitions (s) }\end{array}$} & \multirow[t]{2}{*}{ Yes } & \multirow[t]{2}{*}{ No } \\
\hline & Con: 15 + ; $74.2( \pm 8.1)$ & Con: no vibration & & & \\
\hline \multirow[t]{4}{*}{ Bogaerts et al. [37] } & WBV: $25 \jmath^{\lambda} ; 66.9( \pm 0.7)$ & WBV: squat, deep squat, wide stance & \multirow[t]{2}{*}{ IMVC: knee extension (Nm) } & \multirow[t]{4}{*}{ Yes } & \multirow[t]{4}{*}{ Yes } \\
\hline & Ex: 25 ठ̊; $67.4( \pm 0.9)$ & $\begin{array}{l}\text { squat, toesstand, toes-stand deep, } \\
\text { one-legged squat, and lunge. }\end{array}$ & & & \\
\hline & \multirow[t]{2}{*}{ Con: 32 ô; $68.6( \pm 1.0)$} & $\begin{array}{l}\text { Ex: cardio exercise, strength and } \\
\text { balance training, flexibility exercise }\end{array}$ & \multirow[t]{2}{*}{ RFD: CMJ (cm) on a contact mat. } & & \\
\hline & & Con: no change lifestyle & & & \\
\hline \multirow[t]{3}{*}{ Bogaerts et al. [38] } & WBV: 70; 66.8 & $\begin{array}{l}\text { WBV: exercises for upper and lower } \\
\text { body }\end{array}$ & \multirow[t]{3}{*}{ IMVC: knee extension (Nm) } & \multirow[t]{3}{*}{ Yes } & \multirow[t]{3}{*}{ No } \\
\hline & Ex: 49: 66.8 & $\begin{array}{l}\text { Ex: cardio exercise, strength and } \\
\text { balance training, flexibility exercise }\end{array}$ & & & \\
\hline & $\begin{array}{l}\text { Con: } 61 ; 67.8 \text { (ratio } 01: 9=1.5: 1 \\
\text { for the total sample) }\end{array}$ & Con: no change life style & & & \\
\hline \multirow[t]{4}{*}{ Boegarts [36] } & WBV 1: 26 +; 80.3 ( \pm 5.3$)$ & $\begin{array}{l}\text { WBV: squat, deep squat, wide } \\
\text { stance squat, toes stand and one } \\
\text { legged squat }\end{array}$ & Physiological Profile Assessment [76]: & \multirow[t]{4}{*}{ Yes } & \multirow[t]{4}{*}{ No } \\
\hline & WBV 2: 28 +; $79.8( \pm 5.3)$ & Con 1 \& 2: no change life style & IMVC: knee extension (kg) & & \\
\hline & Con 1: 29 ; $78.7( \pm 5.6)$ & & & & \\
\hline & Con 2: 28 ; $79.6( \pm 5.2)$ & & & & \\
\hline \multirow[t]{3}{*}{ Corrie et al. [65] } & WBV: $21,13+; 81.9( \pm 5.7)$ & $\begin{array}{l}\text { WBV: standing position, with bent } \\
\text { knees and Otago Exercise programm }\end{array}$ & Power: (W/kg body weight) leg press & \multirow[t]{3}{*}{ Yes (power \& CR) } & \multirow[t]{3}{*}{ Yes (power) } \\
\hline & Sham: 20, 16 \%; $79.1( \pm 7.8)$ & Sham: Otago Exercise program which & RFD: CMJ (N/kg body weight & & \\
\hline & & & FS: CR over five repetitions & & \\
\hline
\end{tabular}


Table 1 Study characteristics of the included studies (Continued)

\begin{tabular}{|c|c|c|c|c|c|}
\hline \multirow[t]{2}{*}{$\overline{\text { Gomez-Cabello [42] }}$} & WBV: 24 & WBV: squat position & $\begin{array}{l}\text { Senior Fitness Test battery and Eurofit } \\
\text { Testing Battery [77]: }\end{array}$ & Yes & No \\
\hline & Con: 25 (20 ठ̂, 29 ㅇ) & Con: no change life style & FS: CR over 30 s (repetition) & & \\
\hline \multirow[t]{3}{*}{ Kemmler et al. [44] } & WBV: 50 ㅇ; $68.8( \pm 3.6)$ & $\begin{array}{l}\text { WBV: static and dynamic exercise } \\
\text { (toe stand, squat) }\end{array}$ & IMVC: leg press (N) & Yes & Yes \\
\hline & Ex: 50 +; $68.6( \pm 3.0)$ & $\begin{array}{l}\text { Ex: static and dynamic exercise } \\
\text { (toe stand, squat) without vibration }\end{array}$ & Power: leg press (W/kg) & & \\
\hline & Con: 51 †; $68.1( \pm 2.7)$ & $\begin{array}{l}\text { Con: exercise and relaxation program } \\
\text { once a week (30×60 min) }\end{array}$ & $\begin{array}{l}\text { RFD: leg press (N/ms) \& Squat jump } \\
\text { (jump height, } \mathrm{cm})\end{array}$ & & \\
\hline \multirow[t]{4}{*}{ Kennis et al. [45] } & WBV: $23 \hat{\sigma}$ & WBV: static and dynamic squat, deep & IMVC: at $120^{\circ}$ knee extension (Nm) & Yes & No \\
\hline & Ex: $20 \hat{\jmath}$ & $\begin{array}{l}\text { squat, wide stance squat, 1-legged } \\
\text { squat, lunge, toes-stand, toes-stand } \\
\text { deep, moving heels. }\end{array}$ & & & \\
\hline & Con: $29 \sigma^{\pi}$ & $\begin{array}{l}\text { Ex: } 60-90 \text { min aerobic, resistance, } \\
\text { balance, and flexibility exercises }\end{array}$ & $\begin{array}{l}\text { DS: concentric dynamic knee extension } \\
(\mathrm{Nm}) \text { at a movement velocity at } 120 \% \mathrm{~s} \text {. }\end{array}$ & & \\
\hline & & Con: no change life style & RFD: counter movement jump (high, $\mathrm{cm}$ ) & & \\
\hline \multirow[t]{2}{*}{ Klarner et al. [46] } & WBV: 36 ; ; $68.1( \pm 4.0)$ & WBV: dynamic exercises & IMVC: with leg press $(\mathrm{N})$ & Yes & Yes \\
\hline & Con: 36 \%; $67.6( \pm 4.13)$ & $\begin{array}{l}\text { Con: } 1 / \mathrm{w} \text { low gymnastic exercise } \\
\& \text { relaxation exercise }\end{array}$ & $\begin{array}{l}\text { RFD: with Counter movement jump } \\
(\mathrm{CM} J \text {, jump height, } \mathrm{cm} \text { ) }\end{array}$ & & \\
\hline \multirow[t]{3}{*}{ Lachance [47] } & WBV: 26; $70.4( \pm 7.7)$ & $\begin{array}{l}\text { WBV: static squats }\left(60^{\circ}\right) \text {, lunges }\left(60^{\circ}\right) \\
\text { and heel raises. }\end{array}$ & FS: CR over $30 \mathrm{~s}$ & Yes & No \\
\hline & Ex: 29; $75.9( \pm 7.2)$ & $\begin{array}{l}\text { Ex: static squats }\left(60^{\circ}\right) \text {, lunges }\left(60^{\circ}\right) \\
\text { heel raises, bicep curls, tricep } \\
\text { extensions, }\end{array}$ & & & \\
\hline & $\left(333^{3}, 22\right.$ ㅇ) & $\begin{array}{l}\text { Exercises were progressive in nature } \\
\text { by safely increasing the number of } \\
\text { repetitions completed and/or weight } \\
\text { of the dumbbells. }\end{array}$ & & & \\
\hline \multirow[t]{2}{*}{ Leung et al. [66] } & WBV: 280 q; $74.2( \pm 7.0)$ & $\begin{array}{l}\text { WBV: standing upright without knee } \\
\text { banding }\end{array}$ & IMVC: knee extensor (kg) & Yes & Yes \\
\hline & Con: 316 q; $71.0( \pm 7.0)$ & Non: no change life style & & & \\
\hline \multirow[t]{2}{*}{ Machado et al. [48] } & WBV: 13 ; $79.3( \pm 7.3)$ & $\begin{array}{l}\text { WBV: static and dynamic exercise } \\
\text { (half-squat }\left(120-130^{\circ}\right) \text {, deep squat } \\
\left.\text { (knee angle } 90^{\circ}\right) \text {, a wide-stance squat } \\
\text { and calves. }\end{array}$ & IMVC: leg extensor (N) & Yes & Yes \\
\hline & Con: 13 \%; $76.2( \pm 8.4)$ & Con: no change life style & $\begin{array}{l}\text { Power: output at three relatives loads: } \\
20,40,60 \% \text { of the IMVC. }\end{array}$ & & \\
\hline Mikhael et al. [49] & WBV1: 6,4 ; $63.3( \pm 7.6)$ & $\begin{array}{l}\text { WBV: WBV1 with flexed knees at } 20^{\circ} \\
\text { and WBV } 2 \text { with extended knees. }\end{array}$ & $\begin{array}{l}\text { DS: one repetition maximum }(1 \mathrm{RM}) \text { leg } \\
\text { press }(\mathrm{N}) \text {, relative strength }(\mathrm{kg} / \mathrm{kg}) \text {, leg } \\
\text { press strength }(\mathrm{kg})\end{array}$ & Yes & Yes \\
\hline
\end{tabular}


Table 1 Study characteristics of the included studies (Continued)

\begin{tabular}{|c|c|c|c|c|c|}
\hline & WBV2: 5,3 ; $69.0( \pm 7.6)$ & $\begin{array}{l}\text { Sham: flexed knees at } 20^{\circ} \text { without } \\
\text { vibration }\end{array}$ & $\begin{array}{l}\text { Power: }(\mathrm{W}) \text { and velocity }(\mathrm{cm} / \mathrm{s}) \text { were } \\
\text { measured at } 20,30,40,50,60,70,80 \text {, } \\
90 \text {, and } 100 \% \text { of current } 1 \mathrm{RM} \text {. }\end{array}$ & & \\
\hline & Sham: 8, 4 +; $62.3( \pm 8.8)$ & & FS : CR over $30 \mathrm{~s}$ & & \\
\hline \multirow[t]{3}{*}{ Roelants et al. [53] } & WBV: 30 ; $64.6( \pm 0.7)$ & $\begin{array}{l}\text { WBV: high squat }\left(120^{\circ} \text { and } 130^{\circ} \text {, }\right. \\
\text { deep squat }\left(90^{\circ}\right) \text {, wide-stance } \\
\text { squat and lunge. }\end{array}$ & IMVC: $\left(0^{\circ} / \mathrm{s}\right)$ torque $(\mathrm{Nm})$ of knee extensor & Yes & No \\
\hline & Ex: 30 ; $63.9( \pm 0.8)$ & Ex: resistance exercise & $\begin{array}{l}\text { DS: dynamic extension-flexion movements } \\
\text { (torque: } \mathrm{N} / \mathrm{m} \text { ) between } 90 \text { and } 160^{\circ} \text { at a } \\
\text { velocity of } 50,100 \text { and } 150^{\circ} / \mathrm{s} \text {. }\end{array}$ & & \\
\hline & Con: 29 क; $64.2( \pm 0.6)$ & Con: no change life style & RFD: jump height $(\mathrm{mm})$ on a contact mat & & \\
\hline \multirow[t]{3}{*}{ Sitjà-Rabert et al. [64] } & WBV: $59 ; 64.6( \pm 0.7)$ & WBV: static/dynamic exercises. & FS: CR over five repetitions & Yes & No \\
\hline & Ex: 58; $63.9( \pm 0.8)$ & Ex: static and dynamic exercise & & & \\
\hline & (Total sample $67 \%$ \&) & & & & \\
\hline \multirow[t]{3}{*}{ Verschueren et al. [58] } & WBV: 25 ; $64.6( \pm 3.3)$ & $\begin{array}{l}\text { WBV: static and dynamic knee- } \\
\text { extensor exercises like squat, deep } \\
\text { squat, wide-stance squat, one-legged } \\
\text { squat and lunge. }\end{array}$ & IMVC: knee-extension & Yes & Yes \\
\hline & Ex: 22 ; $63.9( \pm 3.8)$ & $\begin{array}{l}\text { Ex: warm-up, resistance training knee- } \\
\text { extensor on a leg extension and a } \\
\text { leg press machine. Designed to the } \\
\text { guideline of the American College } \\
\text { of Sports Medicine }\end{array}$ & $\begin{array}{l}\text { DS: isokinetic extension-flexion movements } \\
\text { for maximal DS (peak torque } \mathrm{N} / \mathrm{m} \text { ) at a } \\
\text { velocity of } 100^{\circ} / \mathrm{s} \text { between of } 90 \text { and } 160^{\circ} \\
\text { joint angle. }\end{array}$ & & \\
\hline & Con: 24 क; $64.2( \pm 3.1)$ & Con: no change life style & & & \\
\hline \multirow[t]{2}{*}{ Verschueren et al. [57] } & WBV: 28 q; $79.8( \pm 5.3)$ & $\begin{array}{l}\text { WBV: static and dynamic knee- } \\
\text { extensor exercises like squat, deep } \\
\text { squat, wide-stance squat, one-legged } \\
\text { squat and toe-stance. }\end{array}$ & IMVC: Knee-extension (Nm) & Yes & No \\
\hline & Con: 28 P; $79.6( \pm 5.2)$ & Con: no change in life style & DS: Knee-extension (Nm). & & \\
\hline \multicolumn{6}{|c|}{ Sidealternating sinusoidal vibration } \\
\hline \multirow[t]{2}{*}{ Beck et al. [35] } & WBV: 17 ; 68.9 ( \pm 70$)$ & Pos: static with slightly bended knees & \multirow{2}{*}{$\begin{array}{l}\text { FS: wall squat dominant leg }(D L) \text { and non } \\
\text { dominant leg (NDL) \& CR over five } \\
\text { repetitions (s) }\end{array}$} & \multirow[t]{2}{*}{ Yes $=$} & No \\
\hline & Con: 15 q; $74.2( \pm 8.1)$ & Con: no vibration & & & \\
\hline \multirow[t]{2}{*}{ Calder et al. [39] } & \multirow[t]{2}{*}{$\mathrm{N}: 41,30$ O; 80.1} & $\begin{array}{l}\text { WBV: stand with slightly bended knees } \\
\text { ( } 35^{\circ} \text { flexion) \& Physiotherapy }\end{array}$ & \multirow[t]{2}{*}{ FS: CR } & \multirow[t]{2}{*}{ Yes } & No \\
\hline & & Con: Physiotherapy & & & \\
\hline \multirow[t]{2}{*}{ Corrie et al. [65] } & WBV: 21,16 +; $81.9( \pm 5.7)$ & $\begin{array}{l}\text { WBV: standing position, with bent } \\
\text { knees and Otago Exercise }\end{array}$ & Power: (W/kg body weight) leg press & \multirow[t]{2}{*}{ No } & No \\
\hline & Sham: 20, 8 \%; $79.1( \pm 7.8)$ & $\begin{array}{l}\text { Sham: Otago Exercise program which } \\
\text { consisted of } 6 \text { weekly visits }\end{array}$ & RFD: CMJ (N/kg body weight & & \\
\hline
\end{tabular}


Table 1 Study characteristics of the included studies (Continued)

\begin{tabular}{|c|c|c|c|c|c|}
\hline \multirow[t]{6}{*}{ Furness and Maschette [40] } & WBV1: 18 (1/week) & WBV: static with 70 knee flexion & FS: CR & Yes (for WBV2 and WBV3) & No \\
\hline & WBV2: 18 (2/week) & Con: no vibration & & & \\
\hline & WBV3: 19 (3/week) & & & & \\
\hline & Age: $72( \pm 8)$ & & & & \\
\hline & Con: 18 (0/wk) & & & & \\
\hline & 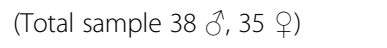 & & & & \\
\hline \multirow[t]{3}{*}{ Furness et al. [41] } & WBV: 19 & WBV: static with $70^{\circ}$ Kneeflexion & FS: CR & Yes & No \\
\hline & Con: 18 & Con: no exercise & & & \\
\hline & (Total sample 16 đ, 21 \&) & & & & \\
\hline \multirow[t]{2}{*}{ Iwamoto et al. [43] } & WBV: 26 ; $72.4( \pm 8.1)$ & WBV: stands with bended knee and hips & FS: CR over 5 times & Yes & No \\
\hline & Con: 26 ; $76.0( \pm 7.4)$ & Con. No exercise & & & \\
\hline \multirow[t]{2}{*}{ Klarner et al. [40] } & WBV: 36 ; $67.9( \pm 3.78)$ & WBV: dynamic exercises & IMVC: Hip \& Knee extension (N) & Yes & Yes \\
\hline & Con: 36 ; $67.6( \pm 4.13)$ & $\begin{array}{l}\text { Con: } 1 / w \text { low gymnastic exercise \& } \\
\text { relaxation exercise }\end{array}$ & RFD: CMJ (jump height, cm) & & \\
\hline \multirow[t]{2}{*}{ Ochi et al. [67] } & WBV: 10 ; $80.9( \pm 2.8)$ & WBV: dynamic exercises & IMVC: Quadiceps muscle dominant leg & Yes & No \\
\hline & Ex: 10 ; $80.2( \pm 3.3)$ & $\begin{array}{l}\text { Con: dynamic exercise: half squat, heel } \\
\text { rise, toe up. }\end{array}$ & & & \\
\hline \multirow[t]{5}{*}{ Raimundo et al. [50] } & WBV: 14 ;; $66( \pm 6)$ & WBV: static with knee angle $120^{\circ}$ & $\begin{array}{l}\text { DS: dynamic maximal unilateral strength } \\
\text { at } 60 \text { and } 300 \% \text { for concentric and } \\
\text { eccentric at } 60 \% \text { (Peak torque }(\mathrm{Nm} / \mathrm{kg} \text { ). }\end{array}$ & Yes & Yes \\
\hline & Ex: 13 \%; $66( \pm 4)$ & Ex: walk-based-programme & Power (W). & & \\
\hline & & Walking over $60 \mathrm{~m}$ with two sets with & RFD: mixed counter movement jump on & & \\
\hline & & $10-15 \%$ of their maximal heart rate. & Ergo Jump Platform (Bosco System, Italy) & & \\
\hline & & & FS: CR over three repetitions. & & \\
\hline \multirow[t]{4}{*}{ Rees et al. [51] } & WBV: 15; $74.3( \pm 5.0)$ & $\begin{array}{l}\text { WBV: static squats over } 4 \text { weeks, than } \\
\text { dynamic squats and calf raises over } \\
4 \text { weeks. }\end{array}$ & $\begin{array}{l}\text { DS: angular velocity } 60 \% \text { for knee and } \\
\text { hip and the angle joint was tested at } \\
30 \% \text { s. }\end{array}$ & Yes & Yes \\
\hline & Ex: $13 ; 73.1( \pm 4.1)$ & Ex: static squats over 4 weeks, than & FS: CR over five repetition & & \\
\hline & Con: $15 ; 73.1( \pm 4.6)$ & $\begin{array}{l}\text { dynamic squats and call raises over } \\
4 \text { weeks, without vibration. }\end{array}$ & & & \\
\hline & (Total sample 23 đ, 20 \&) & & & & \\
\hline \multirow[t]{3}{*}{ Rees et al. [52] } & WBV: $15 ; 74.3( \pm 5.0)$ & $\begin{array}{l}\text { Con: only walking WBV: static and } \\
\text { dynamic exercise (squats, calf raises) }\end{array}$ & DS: as torque $(\mathrm{Nm} / \mathrm{kg})$ & Yes & No \\
\hline & Ex: $13 ; 73.1( \pm 4.1)$ & Ex: same exercise without vibration & \multirow{2}{*}{$\begin{array}{l}\text { maximum isokinetic power (W/kg) angular } \\
\text { velocity for the hip and knee was } 60 \% \mathrm{~s} \text {, } \\
\text { with the ankle joint tested at } 30 \% \mathrm{~s} \text {. }\end{array}$} & & \\
\hline & (No sex distribution information) & & & & \\
\hline
\end{tabular}


Table 1 Study characteristics of the included studies (Continued)

Russo et al. [55] WBV: 17 q $\quad$ WBV: static, knees slightly flexed

Sievänen et al. [61]

Stolzenberg et al. [56]

von Stengel et al. [59]

WBV: 50 ; $68.8( \pm 3.6)$

Zhang et al. [60]
Age: $60.7( \pm 6.1)$

Con: 16 ㅇ

Age: $61.4( \pm 7.3)$

WBV: 8,7 ㅇ

Age: $84.4( \pm 6.3)$

Sham: 7,5 ㅇ

Age: $83.6( \pm 8.9)$

WBV: 30 \%; $67.5( \pm 3.8)$

Con: 30 \%; $65.5( \pm 4.3)$

Ex: 50 q; $68.6( \pm 3.0)$

Con: 51 \%; $68.1( \pm 2.7)$

Con: no change in life style

WBV: dynamic exercise such as slight squatting, toe raises, lateral weigth

transfer.

Ex: light squatting, toe raises or weight transfer forward and lateral weight transfer on WBV.

WBV: static standing with slightly bent knees and hips, continuous squatting from erect standing to $90^{\circ}$ knee flexio or static stance in $90^{\circ}$ knee flexion

Con: balance exercise like Romberg, tandem and single-leg stance.

WBV: heel rise, one-legged deep squat, and leg abduction

Ex: heel rise, one-legged deep squat, and leg abduction without vibration

Con: exercise and relaxation program once a week in blocks of 10 weeks with breaks

WBV: 19, 2 +; 85.8 ( \pm 3.6$)$

Con: 18,3 \% $84.7( \pm 3.7)$
WBV: different to their function. Who could stand: partial squat position with slight hip, knee and ankle joint flexion. Who could not stand

independently, same position, but

were allowed to hold the support

bar with their hands.

Ex: usual care, physical therapy (ultrasound therapy, electrical

stimulation, etc.) and routine

exercises, such as pedalling training

with regular dosage and time of

treatments.
DS: strength (N), acceleration of the

centre of gravity (COG) was calculated

No

as the ratio of force $(\mathrm{N})$ and body mass $(\mathrm{kg})$.

RFD: starting from a standstill, jumped as

high as possible and landed (W).

FS: SPPB

NO

Power: CMJ (W/kg)

RFD: CMJ jump height (cm)

FS: 1-leg hopping and CR over five repetitions

MVC: leg press (N)

Yes

RFD: CMJ (W/Kg)

IMVC: M. quadriceps

Yes

Yes 
Table 1 Study characteristics of the included studies (Continued)

Stochastic resonance vibration

\begin{tabular}{|c|c|c|c|c|c|}
\hline \multirow[t]{3}{*}{ Kessler et al. [23] } & WBV: 10,8 \%; $77( \pm 7.7)$ & \multirow{3}{*}{$\begin{array}{l}\text { static (e.g. normal stance, semi- } \\
\text { tandem, one leg stance) and } \\
\text { dynamic standing (e.g. squat) }\end{array}$} & IMVC: knee-extension (N) & \multirow[t]{3}{*}{ Yes } & \multirow[t]{3}{*}{ Yes } \\
\hline & \multirow[t]{2}{*}{ Sham:10, 8 \%; $81( \pm 5.7)$} & & RFD: knee-extension (N/s) & & \\
\hline & & & FS: SPPB & & \\
\hline \multirow[t]{3}{*}{ Rogan et al. [54] } & WBV: 10; $77( \pm 7.7)$ & \multirow{3}{*}{$\begin{array}{l}\text { static standing with slightly bent } \\
\text { knees and hips }\end{array}$} & \multirow[t]{3}{*}{ FS: CR (1 time) } & \multirow[t]{3}{*}{ Yes } & \multirow[t]{3}{*}{ No } \\
\hline & Sham:10; 81 ( \pm 5.7$)$ & & & & \\
\hline & (No sex distribution information) & & & & \\
\hline \multirow[t]{3}{*}{ Rogan et al. [20] } & WBV: 5; $77( \pm 7.7)$ & \multirow{3}{*}{$\begin{array}{l}\text { static standing with slightly bent } \\
\text { knees and hips }\end{array}$} & IMVC: knee-extension (N) & \multirow[t]{3}{*}{ Yes } & \multirow[t]{3}{*}{ No } \\
\hline & Sham: 4; $81( \pm 5.7)$ & & RFD: knee-extensor (N/s) & & \\
\hline & (Total sample 4 ô, 5 우) & & FS: SPPB & & \\
\hline
\end{tabular}

Abbreviation: Con control group, EX exercise group, Sham sham group; SD standard deviation, GE group effests, mo month, wk week, WBV whole-body vibration, Pos position, $s$ seconds, IMVC isometric maximal

Abbreviation: Con control group, $E X$ exercise group, Sham sham group; $S D$ standard deviation, GE group effests, mo month, wk week, WBV whole-body vibration, POS position, $S$ seconds, IMVC isometric maximal
voluntary contraction, DS dynamic maximal strength, RFD rate of force development, $F S$ functional strength, $C R$ chair rising, CMJ counter movement jump, SPPB Short Physical Performance Battery Test, $C m$ centimetre,

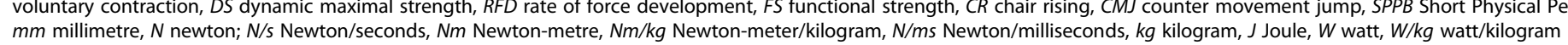


Table 2 Overview of trainings parameter within each study

\begin{tabular}{|c|c|c|c|c|}
\hline Study & Duration/(Session/per week) & Amplitude & Frequency & Sets, Duration, Rest between set \\
\hline \multicolumn{5}{|l|}{ Vertically sinusoidal vibration } \\
\hline Amaral et al. [78] & 12 weeks/(3/week) & $2-4 \mathrm{~mm}$ & $30-40 \mathrm{~Hz}$ & 3 sets $\times 30-45 \mathrm{~s}$. \\
\hline Álvarez- Barbosa et al. [62] & 8 weeks/(3/week) & $4 \mathrm{~mm}$ & $\mathrm{~F}: 30-35 \mathrm{~Hz}$ & 6-12 sets, $12-17$ min total time, $45 \mathrm{~s}$ rest between set \\
\hline Bautmans et al. [34] & 6 weeks/(3/week) & $2-5 \mathrm{~mm}$ & $30-40 \mathrm{~Hz}$ & 4 sets $\times 30-60 \mathrm{~s}, 30-60 \mathrm{~s}$ rest between set \\
\hline Beck et al. [35] & 32 weeks/(2/week) & $0.3 \mathrm{~g}$ & $30 \mathrm{~Hz}$ & 15 min (1 session), no rest \\
\hline Bogaerts et al. [37] & 47 weeks/(3/week) & $2.5-5 \mathrm{~mm}$ & $30-40 \mathrm{~Hz}$ & 4 sets sets $\times 30 s-15 \times 30 s, 15-30 s$ rest between set \\
\hline Bogaerts et al. [38] & 48 weeks $\mathrm{mo} /(3 /$ week $)$ & NA & NA & NA \\
\hline Boegarts [36] & 24 weeks/(3/week) & $1.6-2.2 \mathrm{~g}$ & $30-40 \mathrm{~Hz}$ & 3 sets $\times 15-60 s, 60-5 s$ rest between set \\
\hline Corrie et al. [65] & 12 weeks/(3/week) & $1.3 \mathrm{~mm}$ & $30 \mathrm{~Hz}$ & 3 to 6 sets $\times 20$ to $60 \mathrm{~s}$, $60 \mathrm{~s}$ rest between set \\
\hline Gomez-Cabello [42] & 44 weeks mo/(3/week) & $2 \mathrm{~mm}$ & $40 \mathrm{~Hz}$ & 10 sets $\times 45 \mathrm{~s}, 60$ s rest between set \\
\hline Kemmler et al. [44] & 88 weeks/(2/week) & NA & $25-35 \mathrm{~Hz}$ & NA \\
\hline Kennis et al. [45] & & $2.5-5 \mathrm{~mm}$ & $30-40 \mathrm{~Hz}$ & 4 sets $\times 30$ s till 15 sets $\times 30 s, 15-30$ s rest between set \\
\hline Klarner et al. [46] & 48 weeks mo/(3/week) & & $35 \mathrm{~Hz}$ & 7 sets $\times 90$ s, 40 s rest between set \\
\hline Lachance [47] & 8 weeks/(2/week) & $2 \mathrm{~mm}$ & $35 \mathrm{~Hz}$ & NA \\
\hline Leung et al. [66] & 72 weeks mo/(5/week) & $2 \mathrm{~mm}$ & $35 \mathrm{~Hz}$ & 20 min, rest (NA) \\
\hline Machado et al. [48] & 10 week/(3-5/week) & $2-4 \mathrm{~mm}$ & $20-40 \mathrm{~Hz}$ & $3-8$ sets $\times 30-60$ s, rest $(N A)$ \\
\hline Mikhael et al. [49] & 12 weeks/(3/week) & $1 \mathrm{~mm}$ & $12 \mathrm{~Hz}$ & 10 sets $\times 60 \mathrm{~s}, 60 \mathrm{~s}$ rest between set \\
\hline Roelants et al. [53] & 24 weeks/(3/week) & $2.5-5 \mathrm{~mm}$ & $35-40 \mathrm{~Hz}$ & $1-3$ sets $\times 30-60 \mathrm{~s}$ of one exercise, 60 to $5 \mathrm{~s}$ rest between set \\
\hline Sitjà-Rabert et al. [64] & 6 weeks/(3/week) & $2-4 \mathrm{~mm}$ & $30-35 \mathrm{~Hz}$ & 3 sets $\times 30-60$ s of one exercise, 60 to $5 \mathrm{~s}$ rest between set \\
\hline Verschueren et al. [58] & 24 weeks/(3/week) & $1.7-2.5 \mathrm{~mm}$ & $35-40 \mathrm{~Hz}$ & NA \\
\hline Verschueren et al. [57] & 18 weeks/(3/week) & $1.6-2.2 \mathrm{~g}$ & $30-40 \mathrm{~Hz}$ & $15-60 s \times$ Pos. exercise, $60 s$ till 5 min rest between exercises \\
\hline \multicolumn{5}{|c|}{ Sidealternating sinusoidal vibration } \\
\hline Beck et al. [35] & 32 weeks/(2/week) & $2 \mathrm{~mm}$ & $12.5 \mathrm{~Hz}$ & 2 sets $\times 3 \mathrm{~min}, 60 \mathrm{~s}$ rest between set \\
\hline Calder et al. [39] & 6 weeks & $2 \mathrm{~mm}$ & $20 \mathrm{~Hz}$ & 4 sets $\times 75 \mathrm{~s}, 90 \mathrm{~s}$ rest between set \\
\hline Corrie et al. [65] & 12 weeks/(3/week) & $2.9 \mathrm{~mm}$ & $30 \mathrm{~Hz}$ & 3 to 6 sets $\times 20$ to $60 \mathrm{~s}, 60 \mathrm{~s}$ rest between set \\
\hline Furness and Maschette [40] & 6 weeks & $0.05 \mathrm{~mm}$ & $15-25 \mathrm{~Hz}$ & 5 sets $\times 60 \mathrm{~s}, 60$ s rest between set \\
\hline Furness et al. [41] & 6 weeks/(3/week) & $1 \mathrm{~mm}$ & $15-25 \mathrm{~Hz}$ & 5 sets $\times 60 \mathrm{~s}, 60 \mathrm{~s}$ rest between sets \\
\hline Iwamoto et al. [43] & 18 weeks/(2/week) & NA & $20 \mathrm{~Hz}$ & 4 min, NA rest \\
\hline Klarner et al. [40] & 48 weeks/(3/week) & $3-7 \mathrm{~mm}$ & $12,5 \mathrm{~Hz}$ & 7 sets $\times 90 \mathrm{~s}, 40 \mathrm{~s}$ rest between set \\
\hline Ochi et al. [67] & 12 weeks/(3/week) & $12 \mathrm{~mm}$ & $2,5 \mathrm{~Hz}$ & $180 \mathrm{~s}$, no rest \\
\hline Raimundo et al. [50] & 32 weeks/(3/week) & $6 \mathrm{~mm}$ & $20-30 \mathrm{~Hz}$ & 3 sets $\times 60 \mathrm{~s}, 60 \mathrm{~s}$ rest between set \\
\hline Rees et al. [51] & 8 weeks/(3/week) & $5-8 \mathrm{~mm}$ & $26 \mathrm{~Hz}$ & 6 sets $\times 45$ up to 60 s, $5 \times 45$ up to 80 s rest between set \\
\hline Rees et al. [52] & 8 weeks/(3/week) & $5-8 \mathrm{~mm}$ & $26 \mathrm{~Hz}$ & 6 sets $\times 45-80$ s, $45-80$ s rest between set \\
\hline Russo et al. [55] & 24 weeks/(2/week) & NA & $12-28 \mathrm{~Hz}$ & 3 sets $\times 60-120$ s, 60 s rest between set \\
\hline Sievänen et al. [61] & 10 week/(2/week) & $2-8 \mathrm{~mm}$ & 12 and $18 \mathrm{~Hz}$ & $1-5$ sets $\times 60-120 \mathrm{~s}, 60 \mathrm{~s}$ rest between set \\
\hline Stolzenberg et al. [56] & 36 weeks/(2/week) & NA & $22-26 \mathrm{~Hz}$ & $60-90 \mathrm{~s}$, rest (NA) \\
\hline von Stengel et al. [59] & 74 weeks/(2weeks) & $1.7-2 \mathrm{~mm}$ & $25-35 \mathrm{~Hz}$ & 6 sets $\times 60 \mathrm{~s}, 60$ s rest between set \\
\hline Zhang et al. [60] & 8 weeks/(3-5/week) & $1-3 \mathrm{~mm}$ & $25-35 \mathrm{~Hz}$ & $4-5$ sets $\times 60 s, 60$ s rest between set \\
\hline \multicolumn{5}{|l|}{ Stochastic resonance vibration } \\
\hline Kessler et al. [23] & 4 weeks/(3/week) & - & 3-6 Hz (Noise 4) & 5 sets $\times 60 \mathrm{~s}, 60 \mathrm{~s}$ rest between set \\
\hline Rogan et al. [54] & 4 weeks/(3/week) & - & $5 \mathrm{~Hz}$ (Noise 4) & 5 sets $\times 60 \mathrm{~s}, 60 \mathrm{~s}$ rest between set \\
\hline Rogan et al. [20] & Immediately (acute effects) & & $6 \mathrm{~Hz}$ (Noise 4) & 5 sets $\times 60 s, 60$ s rest between set \\
\hline
\end{tabular}




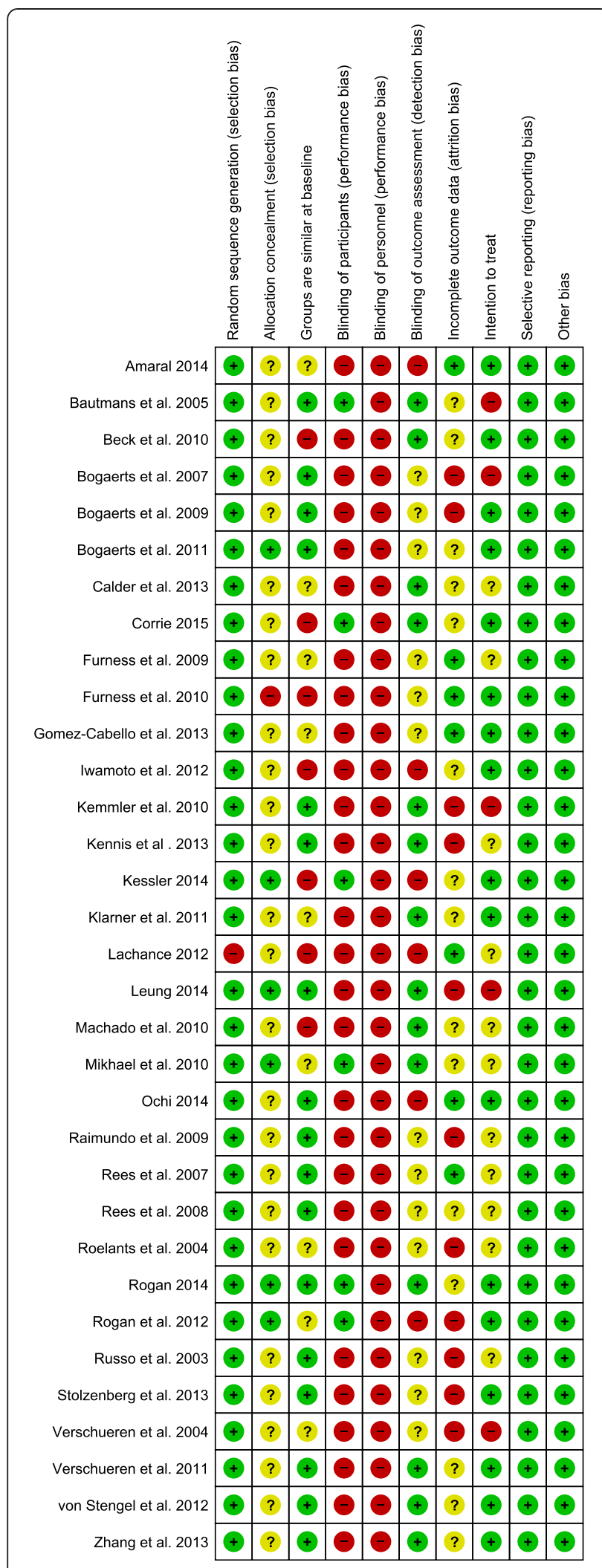

Fig. 2 Risk of bias
No-Go

The subgroup analysis for SS-WBV-No-Go showed a SMD of 0.77 (95\% CI 0.10 to 1.44) in favour of SSWBV.

\section{Dynamic strength: WBV vs. non-exercise control}

Comparing dynamic strength in WBV versus nonexercise controls, six studies [45, 49, 51, 53, 57, 58] contributed with a total of 312 participants. The pooled overall SMD was 0.34 (95\% CI 0.06 to 0.61), which was statistically significant in favour of the WBV group, with low heterogeneity $\left(\mathrm{I}^{2} 26.7 \%, p=0.234\right)$.

\section{Go-Go}

The subgroup analysis for SV-WBV-Go-Go showed a SMD of 0.47 (95\% CI 0.06 to 0.88 ) in favour of SVWBV with a low heterogeneity, $\mathrm{I}^{2} 38.6 \%, p=0.180$. The subgroup analysis for SS-WBV-Go-Go showed a SMD of 0.38 (95\% CI -0.34 to 1.11 ) in favour of SS-WBV.

\section{Slow-Go}

The subgroup analysis for SV-WBV-Slow-Go showed a SMD of 0.09 (95\% CI -0.28 to 0.46 ).

\section{Dynamic strength: WBV vs. conventional exercise}

For the comparison WBV versus conventional exercise seven studies $[45,50-52,58,59,53]$ contributed with a total of 245 participants. The pooled overall SMD was 0.08 (95\% CI -0.34 to 0.17 ), statistically non-significant, thus, not in favour of a particular group, with no heterogeneity $\left(\mathrm{I}^{2} 0.0 \%, p=0.539\right)$.

\section{Go-Go}

The subgroup analysis for SV-WBV-Go-Go showed a SMD of -0.25 (95\% CI -0.59 to 0.08$)$ in favour of exercise, with no heterogeneity, $\mathrm{I}^{2} 0.0 \%(p=0.639)$. The subgroup analysis for SS-WBV-Go-Go showed a SMD of 0.16 (95\% CI -0.27 to 0.60 ) statistically not significant in favour of SS-WBV with no heterogeneity, $\mathrm{I}^{2}$ $0.0 \%(p=0.415)$.

\section{Slow-Go}

The subgroup analysis for SV-WBV-Slow-Go showed a SMD of 0.08 (95\% CI -0.77 to 0.94 ).

\section{Power: WBV vs. non-exercise control}

For the outcome power and the comparison WBV versus non-exercise control five studies $[48,49,55,56,66]$ contributed with a total of 186 participants. The control group of the Corrie et al. [65] study contributed to two subgroup analyses, therefore, we cut the number of participants in the control group in half to avoid incorrect standard errors. 


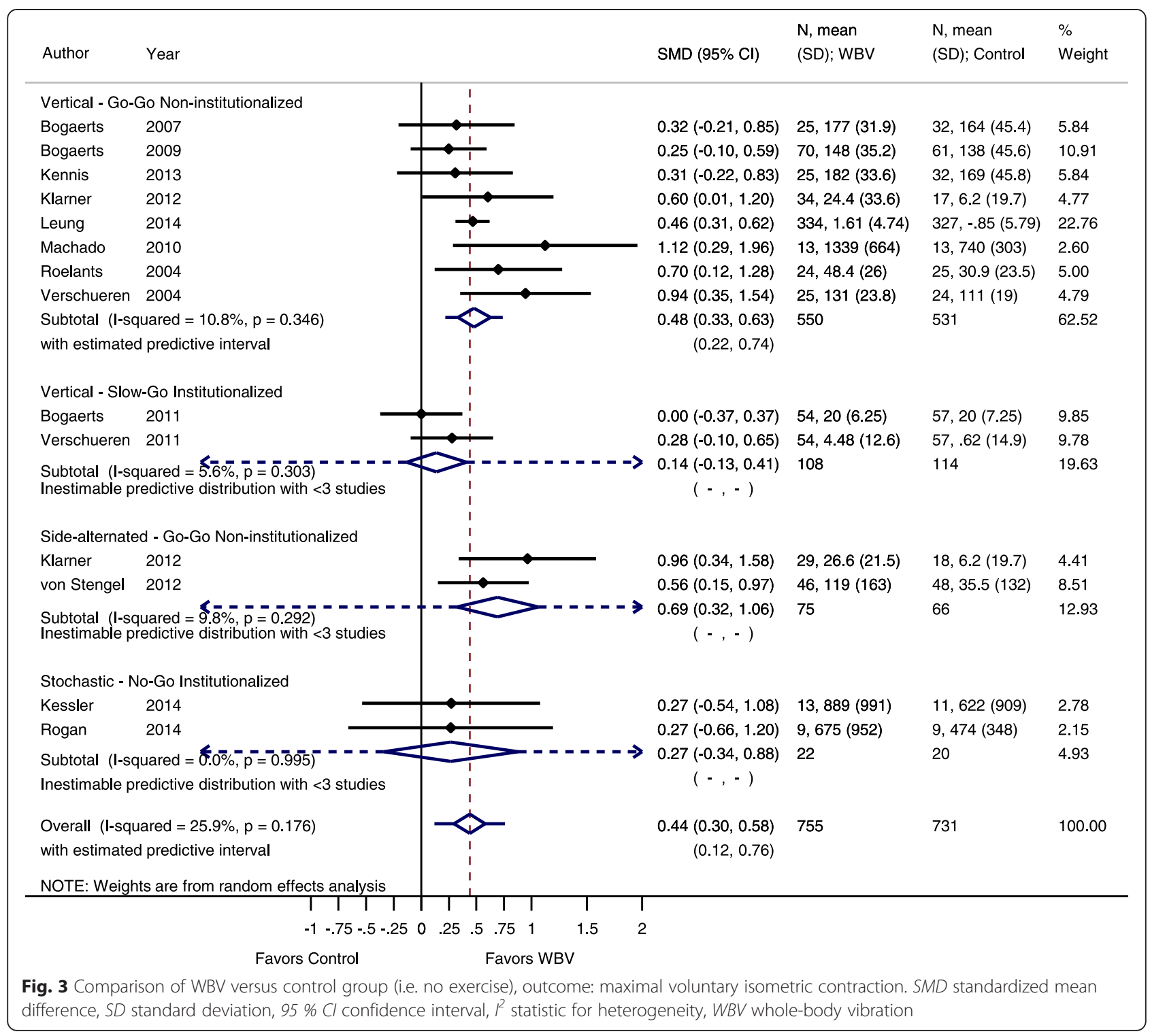

\section{Go-Go}

The pooled overall SMD was 0.22 (95\% CI -0.19 to $0.64)$, statistically non-significant in favour of the WBV group, with a moderate heterogeneity $\left(\mathrm{I}^{2} 44.7 \%\right.$, $p=0.107)$.

The subgroup analysis for SV-WBV-Go-Go showed a SMD of -0.45 (95\% CI -1.14 to 0.25 ), with low heterogeneity, $\mathrm{I}^{2} 17.3 \%(p=0.271)$. SS-WBV-Go-Go showed a SMD of 0.50 (95\% CI 0.07 to 0.92 ) statistically significant in favour of SS-WBV with no heterogeneity, $\mathrm{I}^{2} 0.0 \%(p=0.405)$.

\section{Slow-Go}

The subgroup analysis for SV-WBV-Slow-Go showed a SMD of 0.73 (95\% CI -0.09 to 1.55 ). SS-WBV-Slow-Go showed a SMD of 0.31 (95\% CI -0.49 to 1.12) statistically not significant in favour of SS-WBV.

\section{Power: WBV vs. conventional exercise}

Three studies $[34,50,52]$ with 76 participants contributed to the comparison SS-WBV versus conventional exercise. The SMD was 0.39 (95\% CI -0.11 to $0.90)$, statistically not significant in favour of the WBV group.

\section{Go-Go}

The subgroup analysis for SS-WBV-Go-Go showed a SMD of 0.63 (95\% CI 0.08 to 1.17), statistically significant in favour of SS-WBV with no heterogeneity, $\mathrm{I}^{2} 0.0 \%(p=0.879)$. 


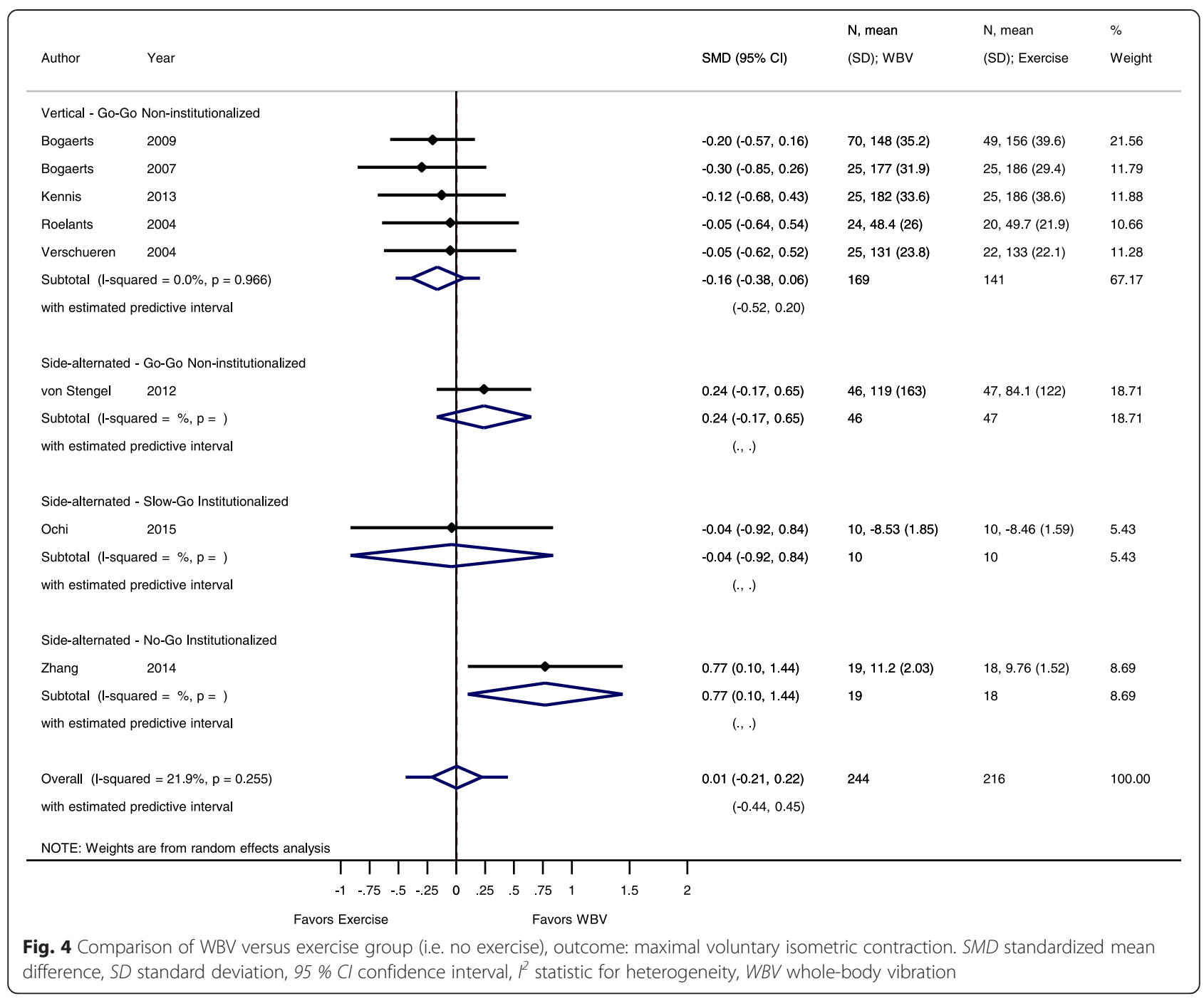

\section{Slow-Go}

The subgroup analysis for SV-WBV-Slow-Go showed a SMD of -0.17 (95\% CI -1.03 to 0.69 ), statistically not significant in favour of conventional exercise.

\section{Rate of force development: WBV vs. non-exercise control}

For the outcome rate of force development and the comparison WBV versus non-exercise control eight studies $[34,37,45,46,55,56,66,53]$ contributed with a total of 500 participants. The control groups of two studies $[46,66]$ contributed to two subgroup analyses, therefore, we divided the number of participants in the control groups in half. The pooled overall SMD was 0.33 (95\% CI 0.14 to 0.52 ), statistically significant in favour of the WBV group with low heterogeneity $\left(\mathrm{I}^{2} 6.1 \%, p=0.385\right)$.

\section{Go-Go}

The subgroup analysis for SV-WBV-Go-Go showed a SMD of 0.39 (95\% CI 0.08 to 0.71 ) in favour of conventional exercise with no heterogeneity, $\mathrm{I}^{2} 0.0 \%$ $(p=0.455)$. SS-WBV-Go-Go showed a SMD of 0.40 (95\% CI 0.16 to 0.64 ) and statistically significant in favour of SS-WBV with no heterogeneity, $\mathrm{I}^{2} 0.0 \%$ $(p=0.516)$.

\section{Slow-Go}

The subgroup analysis for SV-WBV-Slow-Go showed a SMD of -0.45 (95\% CI -1.25 to 0.36 ). The subgroup analysis for SS-WBV-Slow-Go showed a SMD of 0.00 (95\% CI -0.80 to 0.80 ).

Rate of force development: WBV vs. conventional exercise For the comparison WBV versus conventional exercise six studies [34, 37, 45, 50, 59, 53] contributed with a total of 285 participants. The pooled overall SMD was 0.13 (95\% CI -0.11 to 0.36 ), statistically non-significant in favour of the WBV group, with no heterogeneity $\left(\mathrm{I}^{2} 0.0 \%, p=0.452\right)$. 


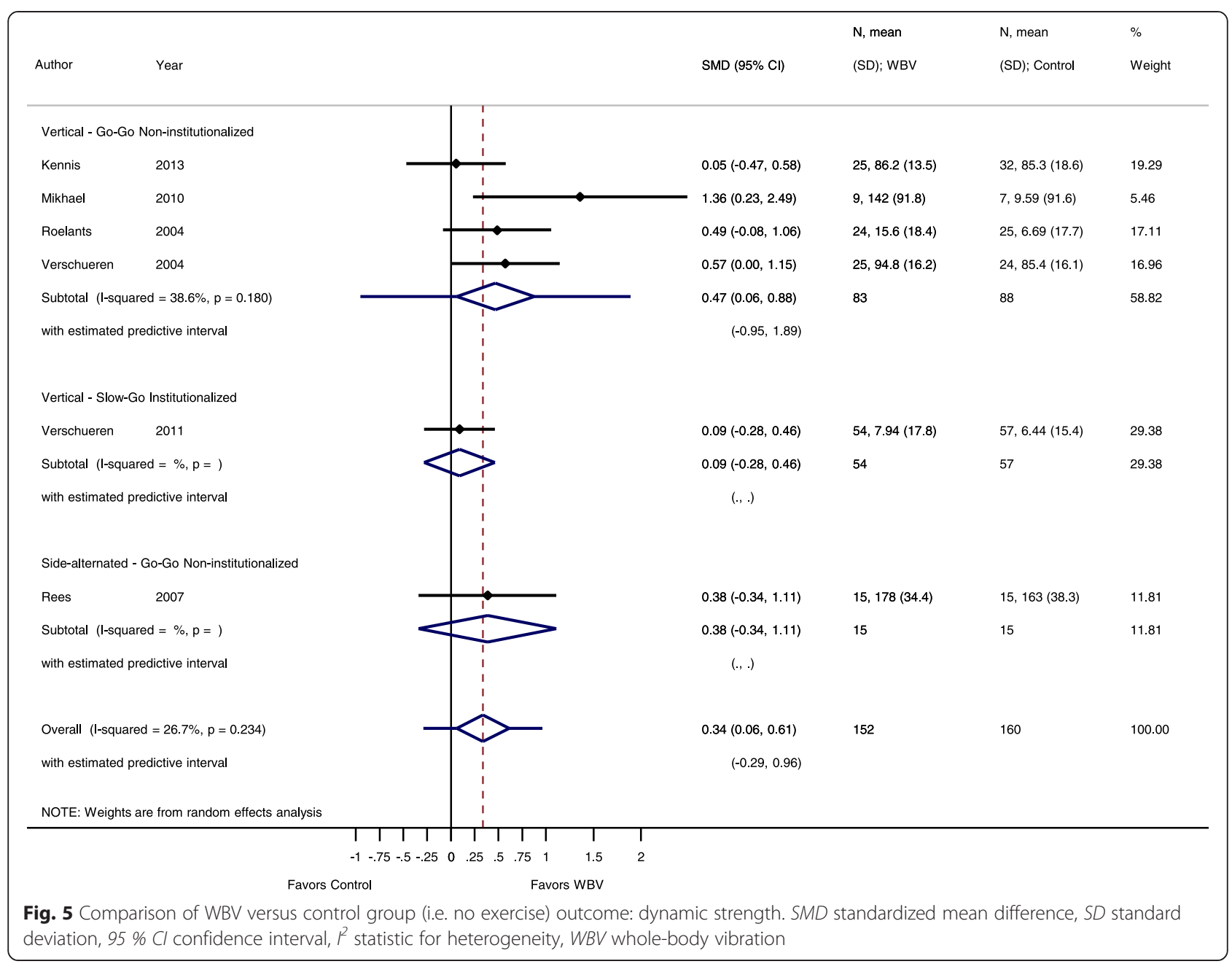

\section{Go-Go}

The subgroup analysis for SV-WBV-Go-Go showed a SMD of -0.06 (95 \% CI -0.39 to 0.26 ) in favour of exercise, with no heterogeneity, $\mathrm{I}^{2} 0.0 \%(p=0.805)$. SSWBV-Go-Go showed a SMD of 0.40 (95\% CI 0.04 to $0.76)$, statistically significant in favour of SS-WBV with no heterogeneity, $\mathrm{I}^{2} 0.0 \%(p=0.405)$.

\section{Slow-Go}

The subgroup analysis for SV-WBV-Slow-Go showed a SMD of -0.10 (95\% CI -0.95 to 0.76$)$.

\section{Functional strength: WBV vs. non-exercise control}

For the outcome functional strength and the comparison WBV versus non-exercise control 17 studies [20, 23, 35, 37, 39-43, 45, 49, 51, 54-56, 59, 64, 53] contributed with a total of 565 participants (one study has SS-WBV and SV-WBV versus control, therefore we cut the number of participants of the control group in half) [35]. The pooled overall SMD was 0.40 (95\% CI 0.20 to 0.60 ), statistically non-significant in favour of the WBV group with low heterogeneity $\left(\mathrm{I}^{2} 24.6 \%, p=0.159\right)$.

\section{Go-Go}

The subgroup analysis for SV-WBV-Go-Go showed an SMD of 0.25 (95\% CI -0.189 to 0.67 ), statistically non-significant in favour of exercise with no heterogeneity, $\mathrm{I}^{2} 0.0 \%(p=0.893)$.SS-WBV-Go-Go showed a SMD of 0.42 (95\% CI 0.13 to 0.71 ) statistically significant in favour of SS-WBV with low heterogeneity, $\mathrm{I}^{2} 27.5 \%(p=0.209)$.

\section{Slow-Go}

The subgroup analysis for SV-WBV-Slow-Go showed an SMD of -0.33 (95\% CI -1.26 to 1.91$)$ with high heterogeneity, $\mathrm{I}^{2} 87.3 \%(p=0.005)$. The subgroup analysis for SS-WBV-Slow-Go showed a SMD of 0.41 (95\% CI -0.40 to 1.21 ), statistically non-significant in favour of SS-WBV with moderate heterogeneity, $\mathrm{I}^{2}$ $58.4 \%(p=0.121)$. 


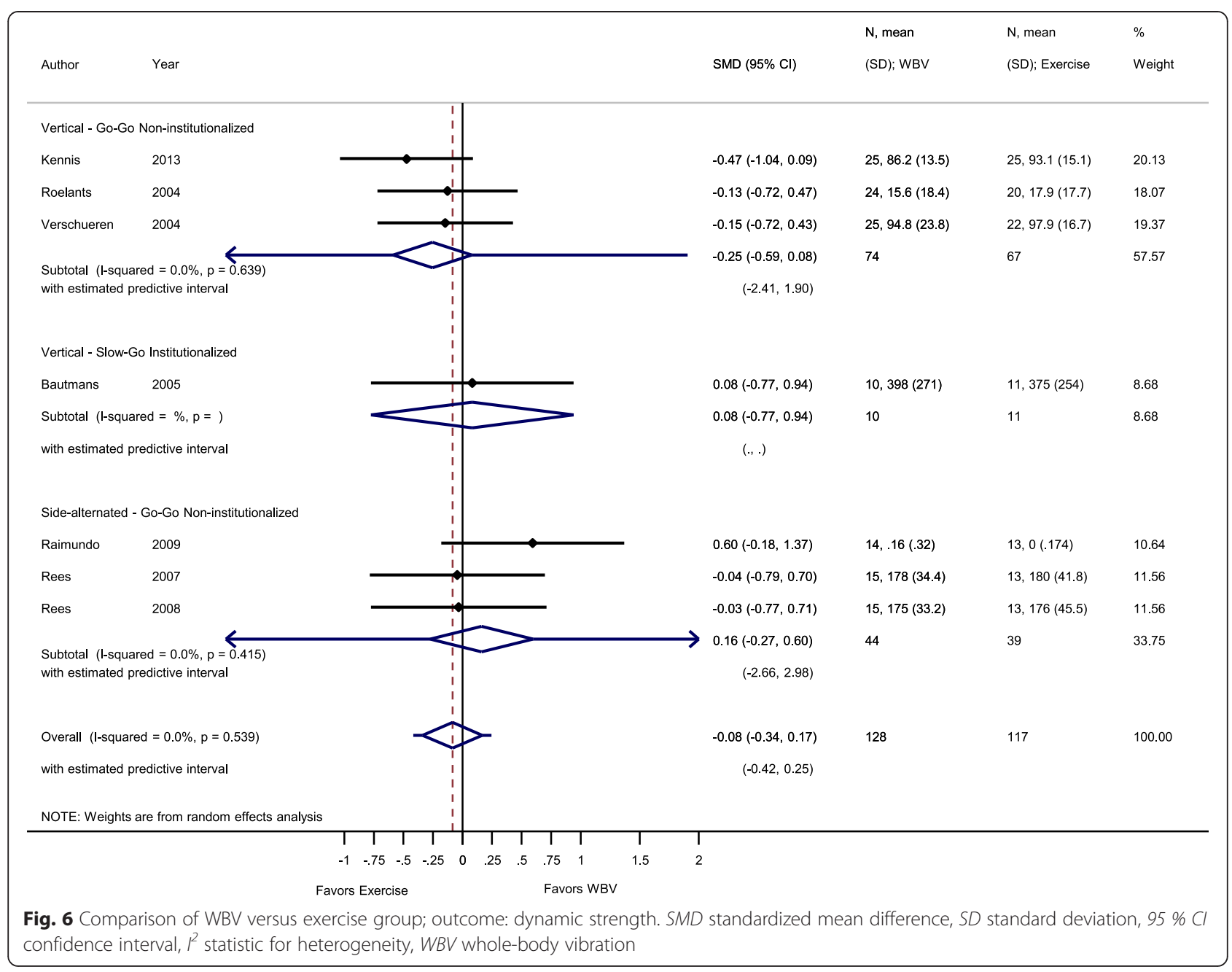

\section{No-Go}

The subgroup analysis for SS-WBV-No-Go showed a SMD of 0.24 (95 \% CI -0.85 to 1.34 ). The subgroup analysis for SR-WBV-Slow-Go showed a SMD of 0.97 (95\% $\mathrm{CI}-0.07$ to 2.00$)$. The subgroup analysis for SR-WBVNo-Go showed a SMD of 0.50 (95\% CI -0.32 to 1.33), statistically non-significant in favour of SR-WBV, with a moderate heterogeneity, $\mathrm{I}^{2} 42.5 \%(p=0.187)$.

\section{Functional strength: WBV vs. conventional exercise}

For the comparison WBV versus conventional exercise five studies $[47,50,51,60,64]$ contributed with a total of 306 participants. The pooled overall SMD was -0.20 (95\% CI -0.67 to 0.27 ), statistically non-significant in favour of the conventional exercise group with substantial heterogeneity $\left(\mathrm{I}^{2} 70.0 \%, p=0.010\right)$.

\section{Go-Go}

The subgroup analysis for SV-WBV-Go-Go showed a SMD of -0.52 (95\% CI -1.06 to 0.02 ), statistically non- significant in favour of conventional exercise. The subgroup analysis for SS-WBV-Go-Go showed a SMD of -0.50 (95\% CI -2.01 to 1.02 ) with high heterogeneity, $\mathrm{I}^{2}$ $86.3 \%(p=0.007)$.

\section{Slow-Go}

The subgroup analysis for SV-WBV-Slow-Go showed a SMD of -0.07 (95\% CI -0.38 to 0.24 ).

\section{No-Go}

The subgroup analysis for SS-WBV-No-Go showed a SMD of 0.44 (95 \% CI -0.21 to 1.09 ), statistically nonsignificant in favour of SS-WBV.

Over all comparisons, only the comparisons of WBV versus non-exercise control for the outcomes isometric strength had a significant Egger's test $(p=0.002)$ for small study bias. Funnel plots for individual comparison are not shown. Figure 13 shows a panel of the funnel plots for all comparisons. 


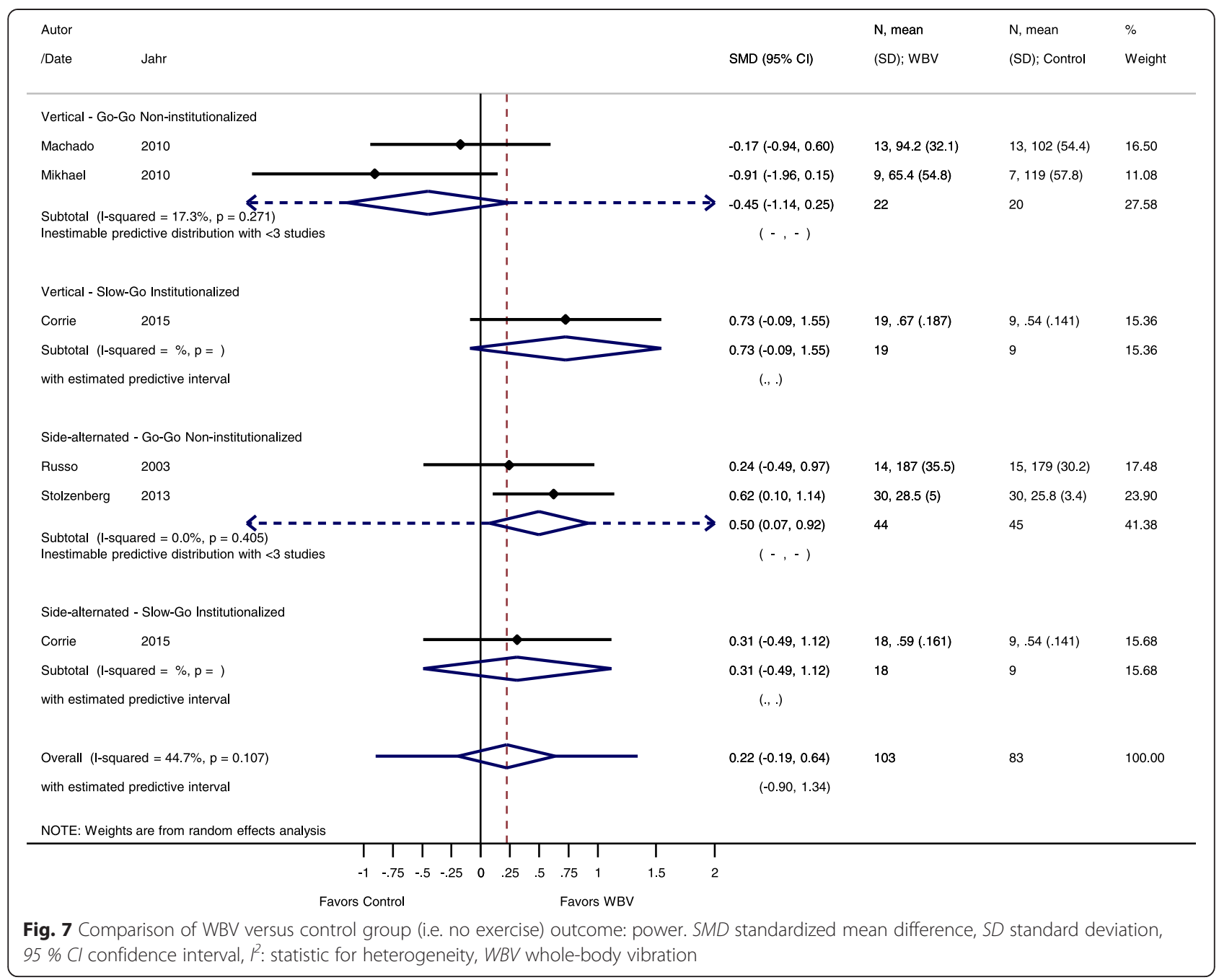

The effect sizes in the studies with participants in the No-Go group (SMD of $0.47,95 \%$ CI 0.16 to $0.78, \mathrm{I}^{2}$ $0.00 \%, p<0.792)$ were higher compared to the Go-Go (SMD 0.26, $95 \%$ CI 0.15 to $0.63, \mathrm{I}^{2} 48.9 \%, p<0.001$ ) and the Slow-Go groups (SMD of 0.14, $95 \%$ CI -0.04 to $0.33, \mathrm{I}^{2} 26.6 \%, p=0.121$ ) (see Table 3 and Additional files 2,3 and 4).

\section{Discussion}

We hypothesized that WBV differently effects on measures of strength and power in Go-Go, Slow-Go and No-Go. This systematic review on muscle-strengthrelated outcomes of WBV in healthy elderly participants included 37 studies in a final analysis, most of which were studies with small sample sizes. The main findings were that WBV showed low to moderate effects in GoGo, Slow-Go and No-Go when compared to nonexercising control groups on proxies of muscle strength in older adults. Furthermore, compared to groups performing more conventional types of exercise, WBV had only small and mostly non-significant advantages. Although only a few studies evaluated the effects of WBV in samples of elderly participants in need of care (classified as No-Go [10] in this review), the highest effect sizes favouring WBV were found in these studies. The few studies that evaluated SR-WBV also resulted in high effect sizes in favour of this type of WBV in NoGo. These findings seem to confirm our hypothesis. However, when we summarized the effect sizes of the meta-analysis and intended to perform statistical analysis on these data that would either refute or confirm our hypothesis, the amount of data was not big enough and the groups too unbalanced in size to allow a credible analysis to be performed. Further studies in No-Go are, therefore, warranted and needed with the various types of WBV. This means regarding our aim to give recommendations on available evidence for practical use the inference must be that at present no recommendations 


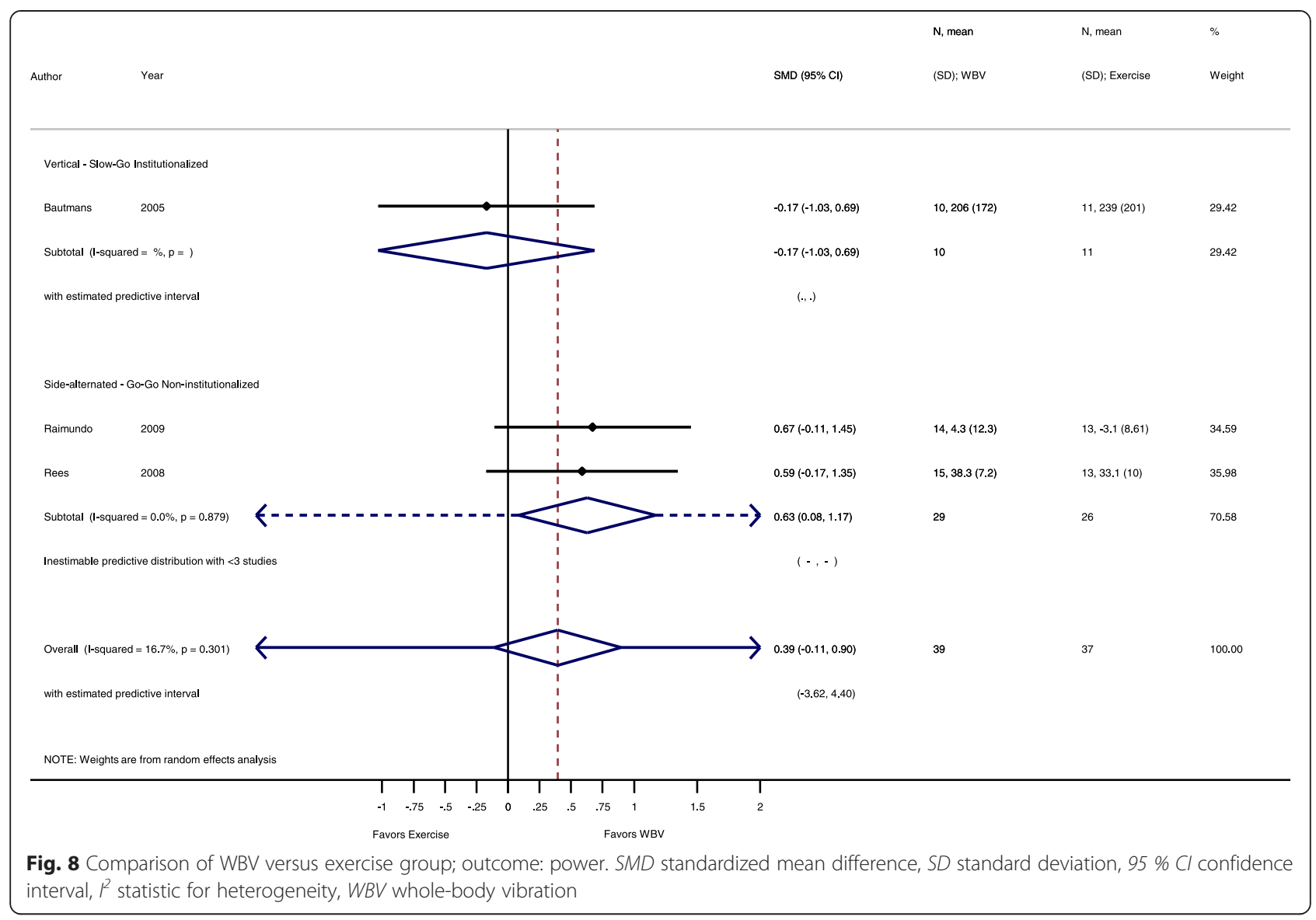

can be given for the most effective vibration mode in elderly persons.

Our review classified the physical capacities of the included participants (i.e. in "Go-Go", "Slow-Go", and "No-Go" [10]) and analysed studies with WBV versus non-exercising control separately from studies comparing WBV versus other types of conventional strength training exercise, as recommended by Orr [68]. Furthermore, we separately analyzed studies using vertical, sidealternating and stochastic resonance WBV. The reason for these distinctions relate to the assumption that initial fitness when entering a training program together with the training content may differently effect on training outcomes. Physical fitness includes health-related (cardiorespiratory endurance, muscular endurance, muscular strength, flexibility and body composition) and skillrelated components (agility, coordination, balance, speed, reaction time and power) [69]. Although exercise recommendations have been published for older adults; e.g. the American College of Sports Medicine (ACSM) [70] guidelines recommend that older adults should undertake $30 \mathrm{~min}$ of moderate intensity, aerobic exercise or activity, five times per week to incur any health benefits, the complex interactions present in various sub-populations of older adults preclude the definition of specific, detailed exercise prescriptions. Furthermore, the number of older people fulfilling the ACSM requirement is rather small and most likely even lower amongst those with low levels of functioning [71]. It seems fair to assume that when principles of exercise training are applied to the development of exercise protocols, clinicians in practical settings can have greater confidence that non-significant research findings reflect deficiencies in exercise efficacy rather than deficiencies in exercise prescription [72]. It is thereby important, however, to consider low baseline fitness and mobility levels in pre-frail or frail or rather untrained elderly when starting an exercise program. Based on the findings of this systematic review it seems that the use of (SR)-WBV is valuable for untrained or frail elderly where the neuromuscular systems might not be able withstanding higher loading and long training sessions, however, with increasing levels of functioning there is a diminishing effect of the WBV interventions. Considering this it becomes clear that this systematic review only reveals first estimates for the possible effect of WBV in (pre-)frail elderly. An important next step would be the design and implementation of a sufficiently powered 


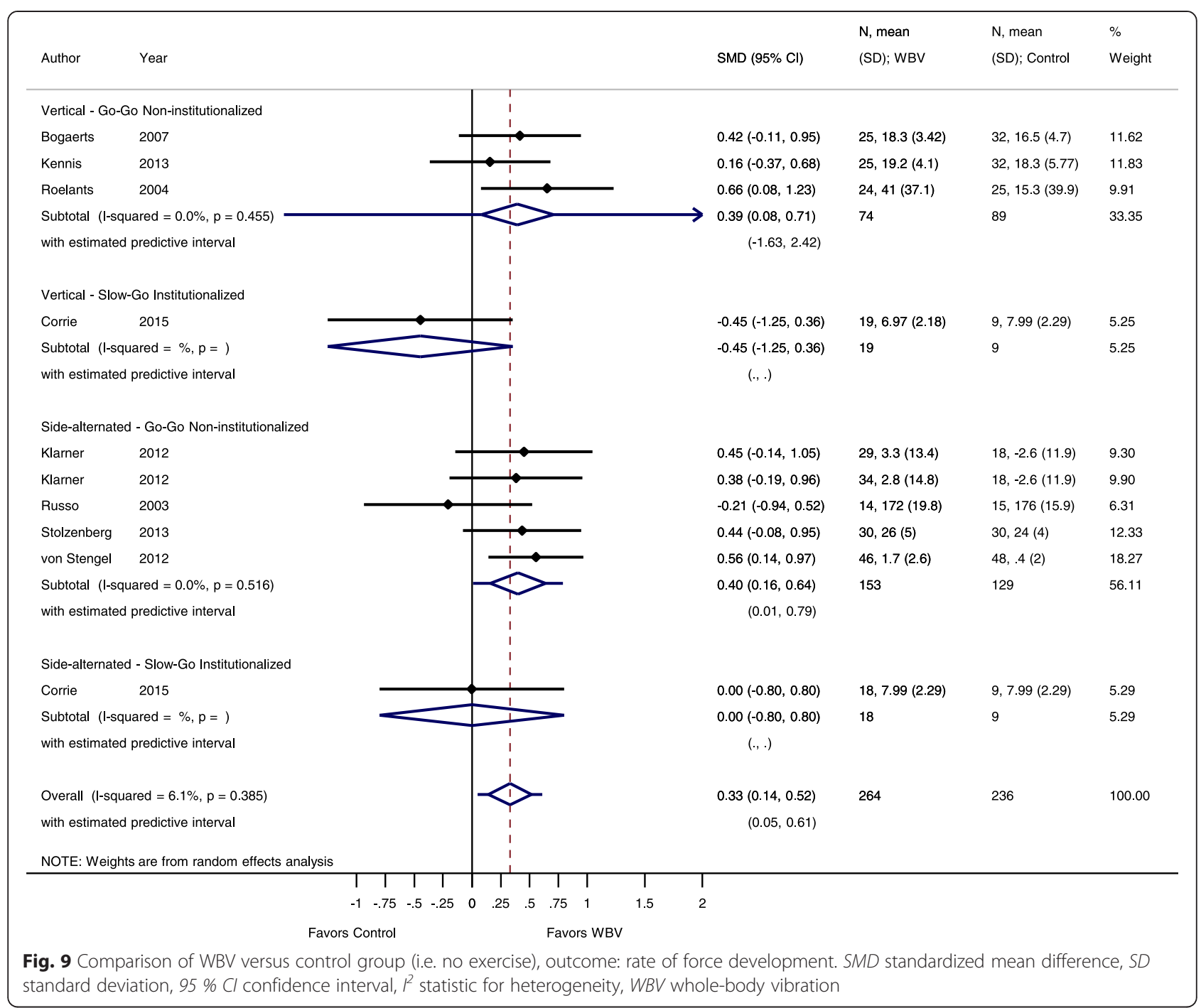

WBV exercise study specifically targeting (pre-)frail institutionalised elderly with a training duration of at least 2 months since this is the duration where effects of WBV training in the elderly may be expected [73].

\section{Role of physical capacity level}

Altough we did not find a systematic review on WBV that used a classification of participants in different subgroups of functioning, our conclusion is in line with similar previously expressed conclusions. Lau et al. [24] mainly focussed on bone mineral density but also included strength measures. They concluded, that WBV is beneficial in elderly persons to increase muscle strength. However, because they did not use a separate analysis for different groups with different levels of physical capacities it is difficult to determine whether the effects observed are dependent on the baseline fitness of the study participants. Sitja-Rabert et al. [25] concluded that WBV was beneficial in elderly participants to improve strength. Osawa et al. [74] included both young and elderly participants and concluded that WBV in addition to exercises or to a normal lifestyle improved knee extensor strength and countermovement jump performance when compared to identical training conditions without WBV.

\section{WBV as skilling-up exercise}

Our findings seem to justify the assumption, that WBV might be applicable as a "skilling-up" exercise for elderly with low physical capacity (i.e. the No-Go group), who are considering to begin with an exercise program, but who are not yet able to perform traditional strength exercises. A short bout of vibration would produce sufficient stimuli to effect on muscle strength, power and functional tasks. There are some theoretical explanations that WBV might improve the neuromuscular 


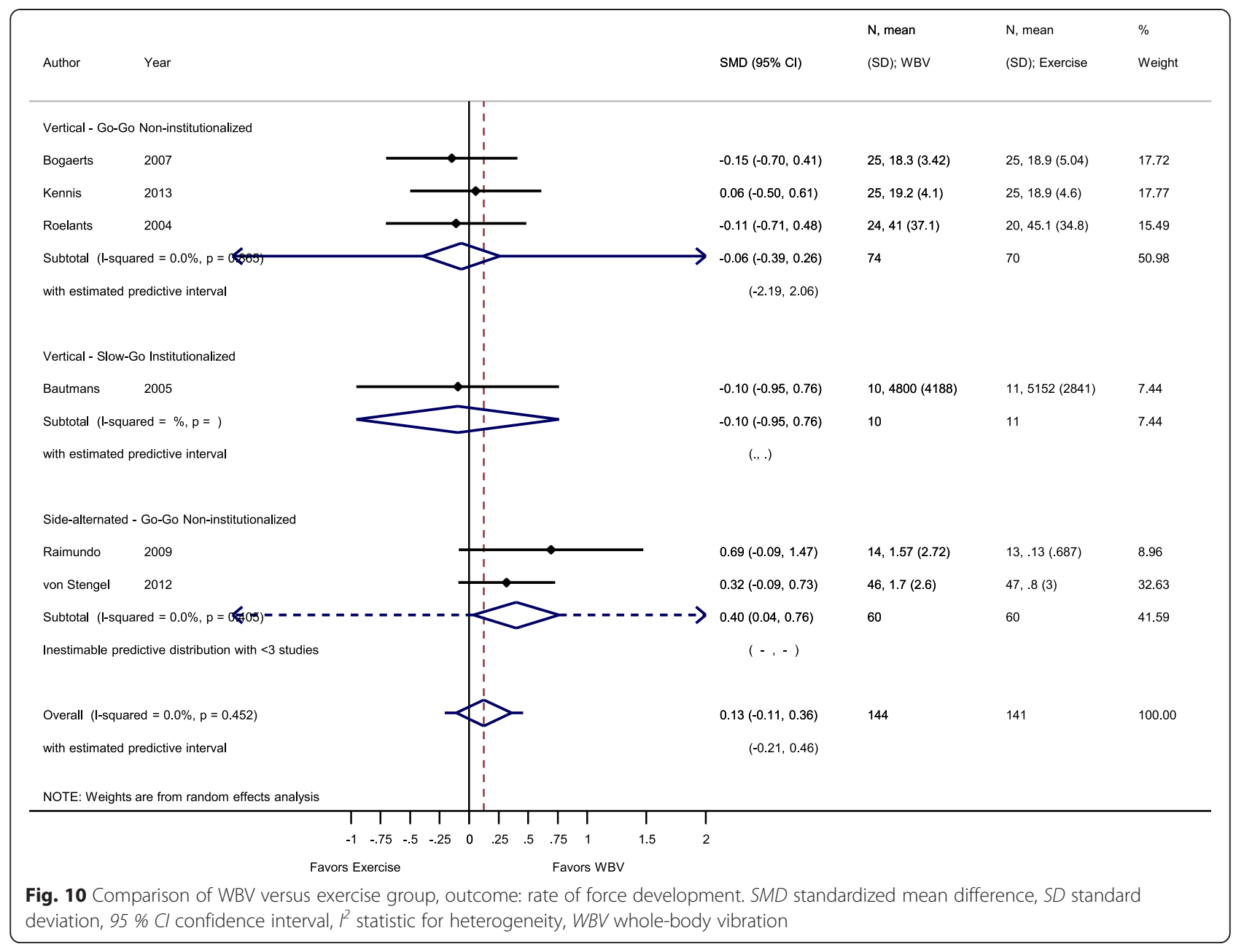

drive, which improves muscular function [18, 75]. In contrary to the No-Go group, elderly persons in the Go-Go and the Slow-Go group can perform standard exercises and WBV might be an additional option among all exercise modalities. The measured effects sizes for the Go-Go and Slow-Go groups were not very high and not all outcomes showed significant differences in the included studies. For this reason, WBV should be rather used for "skilling-up" in pre-frail or frail elderly individuals.

\section{Limitations of this study}

There are some limitations of this review. The included studies presented moderate to high risk of bias, allocation concealment was not described in most studies, groups were often not similar at baseline (which is often the case in small studies), participants were blinded in only seven studies and outcome assessors were blinded in only 11 studies. With the exception of Leung et al. [66], Boegarts et al. [38], Kemmler et al. [44], von Stengel [59] $(n=151)$ and Sitjà-Rabert et al. [64], the remaining studies were small. This further increases the risk of bias. Therefore, the quality of the included studies overall was rather low.

The ability to replicate or reproduce experimental results, or reproducibility, is one of the major tenets of the scientific method. SR-WBV results considered in this review come from one research group only. It is, therefore, necessary that the published findings of this group are validated through replication by others. Until replication is done the results for SRWBV should be interpreted with caution and, hence, this is a limitation of this review. Another limitation of our approach is the possible language bias, we only included studies in English or German and it cannot be excluded that relevant research in different languages exists.

This review included mainly studies with "Go-Go" and "Slow-Go" participants and only few studies with participants from the "No-Go" group. As the effect might be largest in this No-Go group with SMD of 0.47 (95\% CI 0.16 to 0.78 ), further studies should evaluate WBV in 


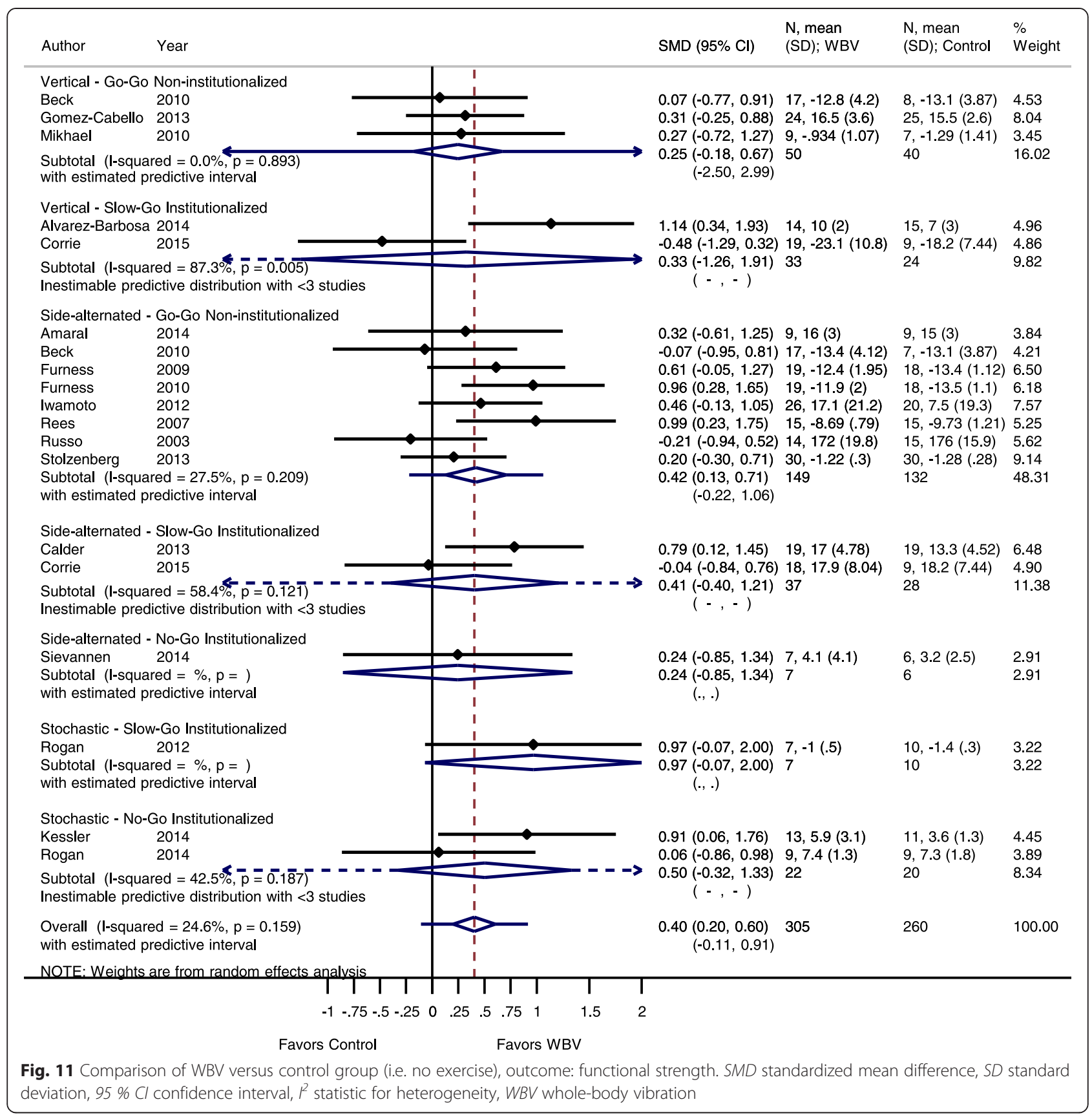

this group of elderly. Only a few studies have evaluated SR-WBV and no study has compared SR-WBV to other vibration modalities in elderly participants.

\section{Conclusions}

WBV shows beneficial effects on proxies of muscle strength in older adults, mainly in elderly with lower initial levels of functioning, suggesting that WBV can be used as a skilling-up exercise in participants. However, the review suggests that WBV has no overall treatment effect on muscle strength properties in older women and men across the whole spectrum of physical functioning. Only few studies evaluated WBV in (pre-)frail elderly. No randomized trial has examined the effects of WBV on muscle in older (pre-)frail elderly. Based on this review no recommendations can be given for the most effective vibration mode. Further studies with the various types of WBV in various sub-populations of elderly persons are warranted and needed to determine the most effective vibration modes. 


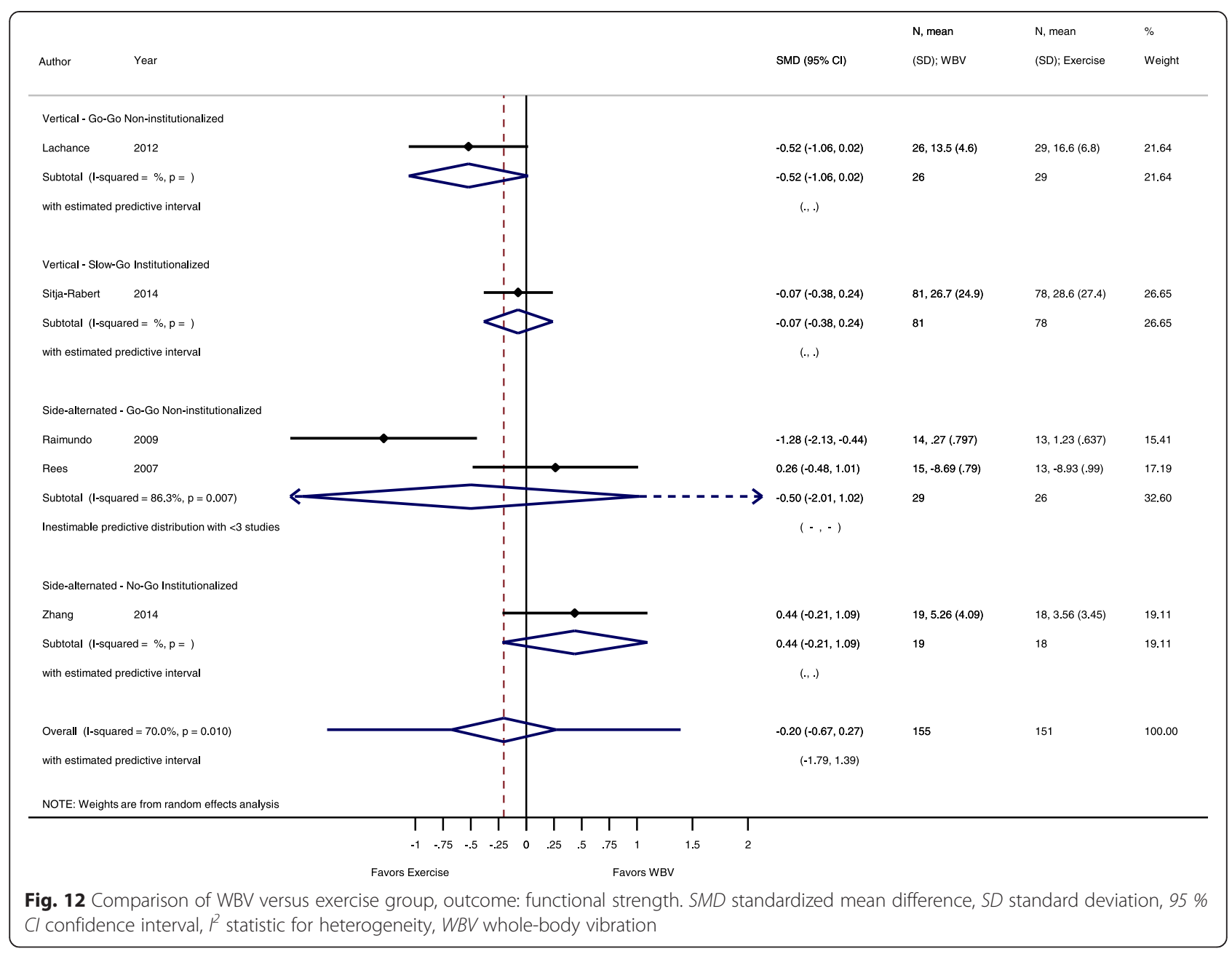

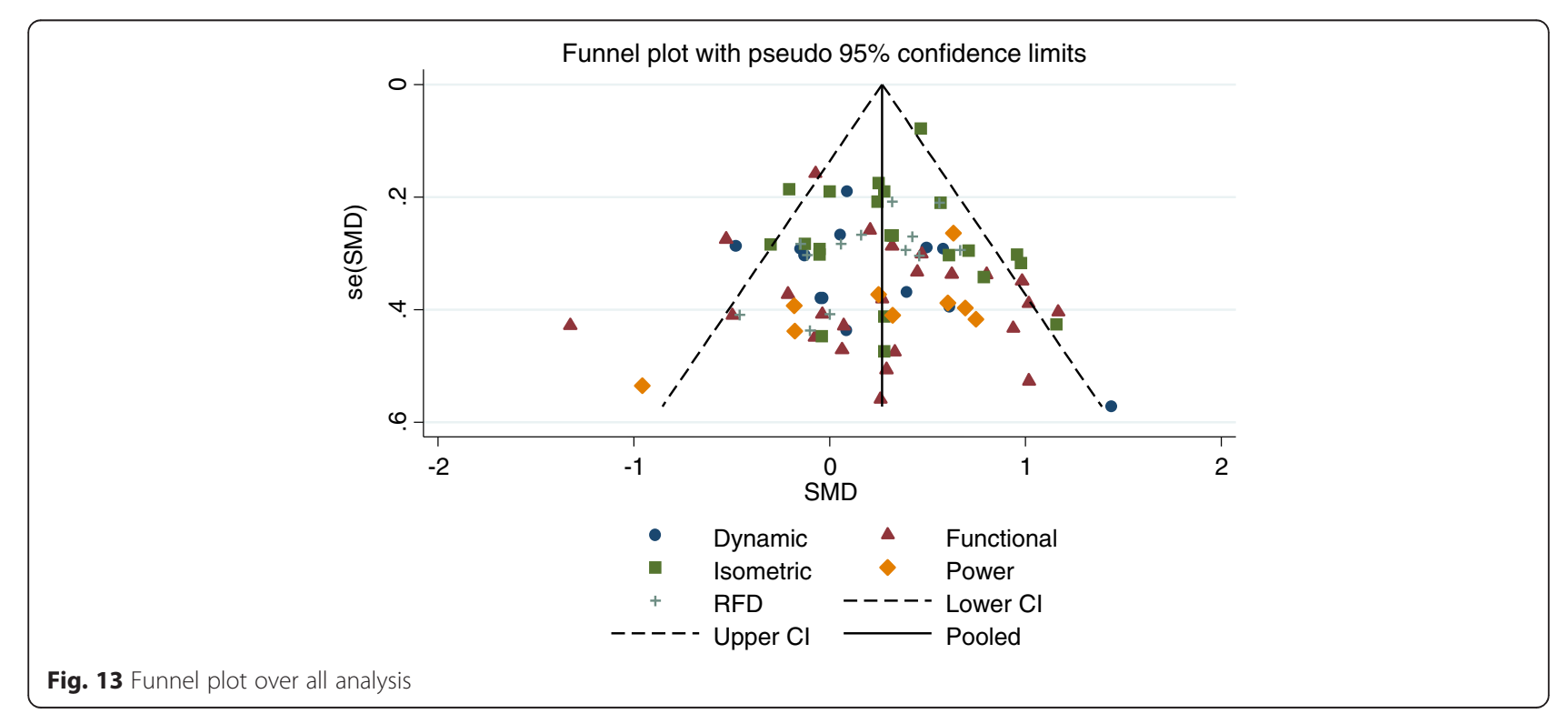


Table 3 Overview of WBV utilization on physical performance status

\begin{tabular}{|c|c|c|c|c|c|}
\hline & Go-Go & & Slow-Go & & No-Go \\
\hline & VS-WBV & & VS-WBV & & \\
\hline 1 & Amaral et al. [78] (SA) FS & 1 & Alvarez et al. [62] FS & & \\
\hline 2 & Beck et al. [35] FS & 2 & Bautmans [34] DS, Power, RFD & & \\
\hline 3 & Boegarts et al. [37] IMVC, RFD & 3 & Boegarts et al. [36] IMVC & & \\
\hline 4 & Boegarts et al. [38] IMVC & 4 & Corrie et al. [6] Power, FS, RFD & & \\
\hline 5 & Gomez-Cabello et al. [42] FS & 5 & Sitja-Rabert et al. [68] FS & & \\
\hline 6 & Kemmler et al. [44] IMVC, Power, & 6 & Verscheuern et al. [57] IMVC, DS & & \\
\hline 7 & RFD & & & & \\
\hline 8 & Kennis et al. [45] IMVC, DS, RFD & & & & \\
\hline 9 & Klarner et al. [46] IMVC, RFD & & & & \\
\hline 10 & Lachane [47] FS & & & & \\
\hline 11 & Leung et al. [66] IMVC & & & & \\
\hline 12 & Machado et al. [48] MVC, Power & & & & \\
\hline 13 & Mikhael et al. [49] DS, Power, FS & & & & \\
\hline 14 & Roelants et al. [53] IMVC, DS, RFD & & & & \\
\hline \multirow[t]{2}{*}{15} & Verscheuren et al. [58] IMVC, DS & & & & \\
\hline & SS-WBV & & SS-WBV & & SS-WBV \\
\hline 1 & Beck et al. [35] FS & 1 & Calder et al. [39] FS & 1 & Sievänen et al. [61] FS \\
\hline 2 & Furness and Maschette [40] FS & 2 & Corrie et al. [65] Power, RFD, FS & 2 & Zhang et al. [60] IMVC, FS \\
\hline 3 & Furness et al. [41] FS & 3 & Ochi et al. [67] IMVC & & \\
\hline 4 & Iwamoto et al. [43] FS & & & & \\
\hline 5 & Klarner et al. [46] IMVC, RFD & & & & \\
\hline 6 & Raimundo et al. [50] DS, Power, RFD; FS & & & & \\
\hline 7 & Rees et al. [51] DS, FS & & & & \\
\hline 8 & Rees et al. [52] DS, Power & & & & \\
\hline 9 & Russo et al. [55] DS, RFD & & & & \\
\hline 10 & Stolzenberg et al. [56] Power, RFD, FS & & & & \\
\hline \multirow[t]{2}{*}{11} & von Stengel et al. [59] IMVC, RFD & & & & \\
\hline & & & SR-WBV & & SR-WBV \\
\hline 1 & & 1 & Rogan et al. [54] FS & 1 & Kessler et al. [23] IMVC, RFD, FS \\
\hline \multirow[t]{3}{*}{2} & & & & 2 & Rogan et al. [20] IMVC, IRFD, FS \\
\hline & SMD 0.26 (95 \% Cl 0.15 to 0.63$)$ & & SMD of 0.14 (95 \% Cl -0.04 to 0.33$)$ & & SMD of 0.47 (95 \% Cl 0.16 to 0.78$)$ \\
\hline & $I^{2} 48.9 \%, p<0.001$ & & $\mathrm{I}^{2} 26.6 \%, p=0.121$ & & $\mathrm{I}^{2} 0.00 \%, p<0.792$ \\
\hline
\end{tabular}

Abbreviation: VS-WBV vertical sinusoidal whole-body vibration, SS-WBV side-alternating whole-body vibration; SR-WBV stochastic resonance whole-body vibration, IMVC isometric maximal voluntary contraction, DS dynamic maximal strength, RFD rate of force development, IRFD isometric rate of force development, FS functional strength, SMD, $I^{2} I^{2}$-statistic for heterogeneity

\section{Additional files}

Additional file 1: Search terms in PubMed. (DOCX $62 \mathrm{~kb}$ )

Additional file 2: Forest plot overview of Classification Go-Go, outcome: all strength outcomes (IMVC, DS, Power, RFD, FS). (PPTX 165 kb)

Additional file 3: Forest plot overview of Classification Slow-Go, outcome: all strength outcomes (IMVC, DS, Power, RFD, FS). (PPTX 105 kb)

Additional file 4: Forest plot overview of Classification No-Go, outcome: all strength outcomes (IMVC, DS, Power, RFD, FS). (PPTX 75 kb)

\section{Abbreviations}

CENTRAL: Cochrane Central Register of Controlled Trials; DS: dynamic strength; FS: functional strength; Go-Go: independent person; IMVC: isometric maximum voluntary contraction; No-Go: person depending permanently on assistance with severe functional limitation; PEDro: Physiotherapy Evidence Database; RFD: rate of force development; Slow-Go: depending on support in everyday activities such as dressing, body care, eating, using the toilet, mobility, and planning the day; SMD: standardised mean differences; SRWBV: stochastic resonance whole-body vibration; SS-WBV: side-alternating sinusoidal whole-body vibration; SV-WBV: sinusoidal vertical whole-body vibration; WBV: whole-body vibration; WHO: World Health Organization. 


\section{Competing interests}

The authors declare no potential conflict of interest with respect to the authorship and/or publication of this article.

\section{Authors' contributions}

SR conceived the idea of this study. $S R, L R$, and $R H$ participated in the conception and design of the study; SR, CJ, CW, NJS, YB, RH collected the data. $\mathrm{RH}$ analysed the data. SR, EDB and $\mathrm{RH}$ wrote the manuscript. EDB, RDB: Methodology, Supervision, critically revising the manuscript for important intellectual content. LR, CJ, CW, NJS, YB, EDB and RH were involved in drafting or revising the manuscript. All authors read and approved the final manuscript.

\section{Authors' information}

-SO Valais-Wallis).

\section{Author details}

${ }^{1}$ Bern University of Applied Sciences, Discipline Physiotherapy, Bern, Switzerland. ${ }^{2}$ Department of Epidemiology, CAPHRI School for Public Health and Primary Care, Maastricht University, PO Box 616, 6200 MD, Maastricht, The Netherlands. ${ }^{3}$ Centre for Evidence Based Physiotherapy, Maastricht University, PO Box 616, 6200 MD, Maastricht, The Netherlands. ${ }^{4}$ Department of Health Sciences and Technology, Institute of Human Movement Sciences and Sport, ETH, Zurich, Switzerland. ${ }^{5}$ School of Health Sciences, University of Applied Sciences and Arts Western, Switzerland, Valais.

Received: 29 April 2015 Accepted: 3 November 2015 Published online: 08 December 2015

\section{References}

1. Aagaard P, Suetta C, Caserotti P, Magnusson SP, Kjaer M. Role of the nervous system in sarcopenia and muscle atrophy with aging: strength training as a countermeasure. Scand J Med Sci Sports. 2010;20(1):49-64.

2. Granacher $U$, Muehlbauer T, Gruber M. A qualitative review of balance and strength performance in healthy older adults: impact for testing and training. J Aging Res. 2012;2012:708905.

3. Manini TM, Clark BC. Dynapenia and aging: an update. J Gerontol A Biol Sci Med Sci. 2012;67(1):28-40.

4. Clark BC, Manini TM. Sarcopenia =/= dynapenia. J Gerontol A Biol Sci Med Sci. 2008;63(8):829-34.

5. Clark BC, Manini TM. What is dynapenia? Nutrition. 2012;28(5):495-503.

6. Clark BC, Manini TM. Functional consequences of sarcopenia and dynapenia in the elderly. Curr Opin Clin Nutr Metab Care. 2010;13(3):271-6.

7. Hughes VA, Frontera WR, Wood M, Evans WJ, Dallal GE, Roubenoff R, et al. Longitudinal muscle strength changes in older adults: influence of muscle mass, physical activity, and health. J Gerontol A Biol Sci Med Sci. 2001;56(5):B209-217.

8. Janssen I. Influence of sarcopenia on the development of physical disability: the Cardiovascular Health Study. J Am Geriatr Soc. 2006;54(1):56-62.

9. de Kort YAW. Housing for the independendtly living elderly; theoretical backgrounds. Eindhoven: Eindhoven University of Technology; 1995

10. Zeyfang A, Braun A. [Guidelines "Diabetes mellitus in the elderly"]. MMW Fortschr Med. 2009;151(20):33-5. 37.

11. Swiss Academy Of M. Treatment and care of elderly persons who are in need of care. Swiss Med Wkly. 2004;134(41-42):618-26.

12. Walter-Ginzburg A, Guralnik JM, Blumstein T, Gindin J, Modan B. Assistance with personal care activities among the old-old in Israel: a national epidemiological study. J Am Geriatr Soc. 2001;49(9):1176-84.

13. Landi F, Marzetti E, Martone AM, Bernabei R, Onder G. Exercise as a remedy for sarcopenia. Curr Opin Clin Nutr Metab Care. 2014;17(1):25-31.

14. Herren K, Holz Hängärtner C, Oberli A, Radlinger L. [Cardiovascular and metabolic strain during stochastic resonance therapy in stroke patients]. Physioscience. 2009;5(1):13-7.

15. Rittweger J, Beller G, Felsenberg D. Acute physiological effects of exhaustive whole-body vibration exercise in man. Clin Physiol. 2000;20(2):134-42.

16. Rittweger J, Ehrig J, Just K, Mutschelknauss M, Kirsch KA, Felsenberg D. Oxygen uptake in whole-body vibration exercise: influence of vibration frequency, amplitude, and external load. Int J Sports Med. 2002;23(6):428-32.
17. Chanou K, Gerodimos V, Karatrantou K, Jamurtas A. Whole-body vibration and rehabilitation of chronic diseases: a review of the literature. J Sports Sci Med. 2012;11(2):187-200

18. Rogan S, Hilfiker R. [Training methods - increase muscle strength due to whole-body vibration - force with Hz]. Sportverletz Sportschaden. 2012; 26(4):185-7.

19. Blasimann A, Fleuti U, Rufener M, Elfering A, Radlinger L. Electromyographic activity of back muscles during stochastic whole body vibration. J Musculoskelet Neuronal Interact. 2014;14(3):311-7.

20. Rogan S, Schmidtbleicher D, Radlinger L. Immediate effects after stochastic resonance whole-body vibration on physical performance on frail elderly for skilling-up training: a blind cross-over randomised pilot study. Aging Clin Exp Res. 2014;26(5):519-27.

21. Nordlund MM, Thorstensson A. Strength training effects of whole-body vibration? Scand J Med Sci Sports. 2007;17(1):12-7.

22. Rittweger J. Vibration as an exercise modality: how it may work, and what its potential might be. Eur J Appl Physiol. 2010;108(5):877-904.

23. Kessler J, Radlinger L, Baur H, Rogan S. Effect of stochastic resonance whole body vibration on functional performance in the frail elderly: a pilot study. Arch Gerontol Geriatr. 2014;59(2):305-11.

24. Lau RW, Liao LR, Yu F, Teo T, Chung RC, Pang MY. The effects of whole body vibration therapy on bone mineral density and leg muscle strength in older adults: a systematic review and meta-analysis. Clin Rehabil. 2011;25(11):975-88.

25. Sitja-Rabert M, Rigau D, Fort Vanmeerghaeghe A, Romero-Rodriguez D, Bonastre Subirana M, Bonfill X. Efficacy of whole body vibration exercise in older people: a systematic review. Disabil Rehabil. 2012;34(11):883-93.

26. Ammann BC, Knols RH, Baschung P, de Bie RA, de Bruin ED. Application of principles of exercise training in sub-acute and chronic stroke survivors: a systematic review. BMC Neurol. 2014;14:167.

27. Liberati A, Altman DG, Tetzlaff J, Mulrow C, Gotzsche PC, loannidis JP, et al. The PRISMA statement for reporting systematic reviews and meta-analyses of studies that evaluate health care interventions: explanation and elaboration. PLoS Med. 2009;6(7):e1000100.

28. Sayers A. Tips and tricks in performing a systematic review-chapter 4. Br J Gen Pract. 2008;58(547):136.

29. American College of Sports Medicine Position Stand. The recommended quantity and quality of exercise for developing and maintaining cardiorespiratory and muscular fitness, and flexibility in healthy adults. Med Sci Sports Exerc. 1998;30(6):975-91.

30. Higgins JP, Altman DG, Gotzsche PC, Juni P, Moher D, Oxman AD, et al. The Cochrane Collaboration's tool for assessing risk of bias in randomised trials. BMJ. 2011:343:d5928.

31. Kakebeeke $\mathrm{TH}$, Knols $\mathrm{RH}$, de Bruin ED. Should rehabilitation specialists use external focus instructions when motor learning is fostered? A systematic review. Sports. 2013;1(2):37-54.

32. Cohen J. Statistical power analysis for the behavioral sciences. 2nd ed. NJ: Erlbaum, Hillsdale; 1988.

33. Higgins J, Thompson S, Deeks J, Altman D. Measuring inconsistency in meta-analysis. BMJ. 2003;327:557-60.

34. Bautmans I, Van Hees E, Lemper JC, Mets T. The feasibility of Whole Body Vibration in institutionalised elderly persons and its influence on muscle performance, balance and mobility: a randomised controlled trial [ISRCTN62535013]. BMC Geriatr. 2005;5:17.

35. Beck BR, Norling TL. The effect of 8 mos of twice-weekly low- or higher intensity whole body vibration on risk factors for postmenopausal hip fracture. Am J Phys Med Rehabil. 2010;89(12):997-1009.

36. Bogaerts A, Delecluse C, Boonen S, Claessens AL, Milisen K, Verschueren SM. Changes in balance, functional performance and fall risk following whole body vibration training and vitamin D supplementation in institutionalized elderly women. A 6 month randomized controlled trial. Gait Posture. 2011;33(3):466-72.

37. Bogaerts A, Delecluse C, Claessens AL, Coudyzer W, Boonen S, Verschueren SM. Impact of whole-body vibration training versus fitness training on muscle strength and muscle mass in older men: a 1-year randomized controlled trial. J Gerontol A Biol Sci Med Sci. 2007:62(6):630-5.

38. Bogaerts AC, Delecluse C, Claessens AL, Troosters T, Boonen S, Verschueren SM. Effects of whole body vibration training on cardiorespiratory fitness and muscle strength in older individuals (a 1-year randomised controlled trial). Age Ageing. 2009;38(4):448-54.

39. Calder CG, Mannion J, Metcalf PA. Low-intensity whole-body vibration training to reduce fall risk in active, elderly residents of a retirement village. J Am Geriatr Soc. 2013;61(8):1424-6. 
40. Furness TP, Maschette WE. Influence of whole body vibration platform frequency on neuromuscular performance of community-dwelling older adults. J Strength Cond Res. 2009;23(5):1508-13.

41. Furness TP, Maschette WE, Lorenzen C, Naughton GA, Williams MD. Efficacy of a whole-body vibration intervention on functional performance of community-dwelling older adults. J Altern Complement Med. 2010;16(7):795-7.

42. Gomez-Cabello A, Gonzalez-Aguero A, Ara I, Casajus JA, Vicente-Rodriguez $\mathrm{G}$. Effects of a short-term whole body vibration intervention on physical fitness in elderly people. Maturitas. 2013;74(3):276-8.

43. Iwamoto J, Sato Y, Takeda T, Matsumoto H. Whole body vibration exercise improves body balance and walking velocity in postmenopausal osteoporotic women treated with alendronate: Galileo and Alendronate Intervention Trail (GAIT). J Musculoskelet Neuronal Interact. 2012;12(3):136-43.

44. Kemmler W, VS S, Mayer S, Niedermayer M, Hentschke C, Kalender WA. [Effect of whole body vibration on the neuromuscular performance of females 65 years and older. One-year results of the controlled randomized ELVIS study]. Z Gerontol Geriatr. 2010;43(2):125-32.

45. Kennis E, Verschueren SM, Bogaerts A, Coudyzer W, Boonen S, Delecluse $C$. Effects of fitness and vibration training on muscle quality: a 1-year postintervention follow-up in older men. Arch Phys Med Rehabil. 2013;94(5):910-8.

46. Klarner A, von Stengel S, Kemmler W, Kladny B, Kalender W. [Effects of two different types of whole body vibration on neuromuscular performance and body composition in postmenopausal women]. Dtsch Med Wochenschr. 2011;136(42):2133-9.

47. Lachane CC. The effects of whole-body vibration exercise on muscular strength in seniors. Ontario: University of Windsor; 2012.

48. Machado A, Garcia-Lopez D, Gonzalez-Gallego J, Garatachea N. Whole-body vibration training increases muscle strength and mass in older women: a randomized-controlled trial. Scand J Med Sci Sports. 2010;20(2):200-7.

49. Mikhael M, Orr R, Amsen F, Greene D, Singh MA. Effect of standing posture during whole body vibration training on muscle morphology and function in older adults: a randomised controlled trial. BMC Geriatr. 2010;10:74.

50. Raimundo AM, Gusi N, Tomas-Carus P. Fitness efficacy of vibratory exercise compared to walking in postmenopausal women. Eur J Appl Physiol. 2009;106(5):741-8.

51. Rees S, Murphy A, Watsford M. Effects of vibration exercise on muscle performance and mobility in an older population. J Aging Phys Act. 2007; 15(4):367-81.

52. Rees S, Murphy A, Watsford ML. Effects of whole-body vibration exercise on lower-extremety muscle strength and power in an older population: a randomized clinical trial. Phys Ther. 2008;88:462-70.

53. Roelants M, Delecluse C, Verschueren SM. Whole-body-vibration training increases knee-extension strength and speed of movement in older women. J Am Geriatr Soc. 2004;52(6):901-8.

54. Rogan S, Hilfiker R, Schmid S, Radlinger L. Stochastic resonance whole-body vibration training for chair rising performance on untrained elderly: a pilot study. Arch Gerontol Geriatr. 2012;55(2):468-73.

55. Russo CR, Lauretani F, Bandinelli S, Bartali B, Cavazzini C, Guralnik JM, et al. High-frequency vibration training increases muscle power in postmenopausal women. Arch Phys Med Rehabil. 2003;84(12):1854-7.

56. Stolzenberg N, Belavy DL, Rawer R, Felsenberg D. Vibration or balance training on neuromuscular performance in osteopenic women. Int J Sports Med. 2013;34(11):956-62.

57. Verschueren SM, Bogaerts A, Delecluse C, Claessens AL, Haentjens P, Vanderschueren $D$, et al. The effects of whole-body vibration training and vitamin D supplementation on muscle strength, muscle mass, and bone density in institutionalized elderly women: a 6-month randomized, controlled trial. J Bone Miner Res. 2011;26(1):42-9.

58. Verschueren SM, Roelants M, Delecluse C, Swinnen S, Vanderschueren D, Boonen S. Effect of 6-month whole body vibration training on hip density, muscle strength, and postural control in postmenopausal women: a randomized controlled pilot study. J Bone Miner Res. 2004;19(3):352-9.

59. von Stengel S, Kemmler W, Engelke K, Kalender WA. Effect of whole-body vibration on neuromuscular performance and body composition for females 65 years and older: a randomized-controlled trial. Scand J Med Sci Sports. 2012;22(1):119-27.

60. Zhang L, Weng C, Liu M, Wang Q, Liu L, He Y. Effect of whole-body vibration exercise on mobility, balance ability and general health status in frail elderly patients: a pilot randomized controlled trial. Clin Rehabil. 2014;28(1):59-68.

61. Sievanen H, Karinkanta S, Moisio-Vilenius P, Ripsaluoma J. Feasibility of whole-body vibration training in nursing home residents with low physical function: a pilot study. Aging Clin Exp Res. 2014;26(5):511-7.

62. Alvarez-Barbosa F, Del Pozo-Cruz J, Del Pozo-Cruz B, Alfonso-Rosa RM, Rogers ME, Zhang Y. Effects of supervised whole body vibration exercise on fall risk factors, functional dependence and health-related quality of life in nursing home residents aged 80+. Maturitas. 2014;79(4):456-63.

63. Osugi T, Iwamoto J, Yamazaki M, Takakuwa M. Effect of a combination of whole body vibration exercise and squat training on body balance, muscle power, and walking ability in the elderly. Ther Clin Risk Manag. 2014;10:131-8.

64. Sitja-Rabert M, Martinez-Zapata MJ, Fort Vanmeerhaeghe A, Rey Abella F Romero-Rodriguez D, Bonfill X. Effects of a Whole Body Vibration (WBV) exercise intervention for institutionalized older people: a randomized, multicentre, parallel, clinical trial. J Am Med Dir Assoc. 2015;16(2):125-31.

65. Corrie H, Brooke-Wavell K, Mansfield NJ, Cowley A, Morris R, Masud T. Effects of vertical and side-alternating vibration training on fall risk factors and bone turnover in older people at risk of falls. Age Ageing. 2015;44(1):115-22.

66. Leung KS, Li CY, Tse YK, Choy TK, Leung PC, Hung W, et al. Effects of 18month low-magnitude high-frequency vibration on fall rate and fracture risks in 710 community elderly - a cluster-randomized controlled trial. Osteoporos Int. 2014;25(6):1785-95.

67. Ochi A, Abe T, Yamada K, Ibuki S, Tateuchi H, Ichihashi N. Effect of balance exercise in combination with whole-body vibration on muscle activity of the stepping limb during a forward fall in older women: a randomized controlled pilot study. Arch Gerontol Geriatr. 2015;60(2):244-51.

68. Orr R. The effect of whole body vibration exposure on balance and functional mobility in older adults: a systematic review and meta-analysis. Maturitas. 2015;80(4):342-58.

69. Caspersen CJ, Powell KE, Christenson GM. Physical activity, exercise, and physical fitness: definitions and distinctions for health-related research. Public Health Rep. 1985;100(2):126-31.

70. Medicine ACOS. ACSM's guidelines for exercise testing and prescription. Baltimore, Philadelphia (US): ACSM (Lippincott Raven); 2013. See: https:// www.lww.com/product/9781609139551

71. Forster A, Lambley R, Young JB. Is physical rehabilitation for older people in long-term care effective? Findings from a systematic review. Age Ageing 2010;39(2):169-75.

72. Campbell KL, Neil SE, Winters-Stone KM. Review of exercise studies in breast cancer survivors: attention to principles of exercise training. Br J Sports Med. 2012;46(13):909-16.

73. Kawanabe K, Kawashima A, Sashimoto I, Takeda T, Sato Y, Iwamoto J. Effect of whole-body vibration exercise and muscle strengthening, balance, and walking exercises on walking ability in the elderly. Keio J Med. 2007;56(1):28-33.

74. Osawa Y, Oguma Y, Ishii N. The effects of whole-body vibration on muscle strength and power: a meta-analysis. J Musculoskelet Neuronal Interact. 2013;13(3):380-90.

75. Cardinale $\mathrm{M}$, Bosco $\mathrm{C}$. The use of vibration as an exercise intervention. Exerc Sport Sci Rev. 2003;31(1):3-7.

76. Lord SR, Menz HB, Tiedemann A. A physiological profile approach to falls risk assessment and prevention. Phys Ther. 2003;83(3):237-52.

77. Pedrero-Chamizo R, Gomez-Cabello A, Delgado S, Rodriguez-Llarena S, Rodriguez-Marroyo JA, Cabanillas E, et al. Physical fitness levels among independent non-institutionalized Spanish elderly: the elderly EXERNET multi-center study. Arch Gerontol Geriatr. 2012;55(2):406-16.

78. Amaral PC, Rica RL, Evangelista AL, Casarin CAS, Junior JAS, Bocalini DS. Whole-body vibration training does not modify anthropometric parameters and lower limb strength in elderly people. Med Sci Tech. 2014;55:6-10. 